This item was submitted to Loughborough's Research Repository by the author.

Items in Figshare are protected by copyright, with all rights reserved, unless otherwise indicated.

\title{
Simulation of all-scale atmospheric dynamics on unstructured meshes
}

PLEASE CITE THE PUBLISHED VERSION

http://dx.doi.org/10.1016/j.jcp.2016.06.048

\section{PUBLISHER}

(c) Elsevier

\section{VERSION}

AM (Accepted Manuscript)

\section{PUBLISHER STATEMENT}

This work is made available according to the conditions of the Creative Commons Attribution-NonCommercialNoDerivatives 4.0 International (CC BY-NC-ND 4.0) licence. Full details of this licence are available at: https://creativecommons.org/licenses/by-nc-nd/4.0/

\section{LICENCE}

CC BY-NC-ND 4.0

\section{REPOSITORY RECORD}

Smolarkiewicz, Piotr K., Joanna Szmelter, and Feng Xiao. 2016. "Simulation of All-scale Atmospheric Dynamics on Unstructured Meshes”. Loughborough University. https://hdl.handle.net/2134/23201. 


\title{
Simulation of all-scale atmospheric dynamics on unstructured meshes
}

\author{
Piotr K. Smolarkiewicz ${ }^{\mathrm{a}}$, Joanna Szmelter ${ }^{\mathrm{b}}$, and Feng Xiao ${ }^{\mathrm{c}}$ \\ ${ }^{a}$ European Centre For Medium Range Weather Forecasts, Reading, RG2 9AX, UK \\ ${ }^{\mathrm{b}}$ Loughborough University, Leicestershire, LE11 3TU, UK \\ ${ }^{\mathrm{c}}$ Tokyo Institute of Technology, Yokohama, 226-8502, Japan
}

\begin{abstract}
The advance of massively parallel computing in the nineteen nineties and beyond encouraged finer grid intervals in numerical weather-prediction models. This has improved resolution of weather systems and enhanced the accuracy of forecasts, while setting the trend for development of unified all-scale atmospheric models. This paper first outlines the historical background to a wide range of numerical methods advanced in the process. Next, the trend is illustrated with a technical review of a versatile nonoscillatory forward-in-time finite-volume (NFTFV) approach, proven effective in simulations of atmospheric flows from small-scale dynamics to global circulations and climate. The outlined approach exploits the synergy of two specific ingredients: the MPDATA methods for the simulation of fluid flows based on the sign-preserving properties of upstream differencing; and the flexible finite-volume median-dual unstructured-mesh discretisation of the spatial differential operators comprising PDEs of atmospheric dynamics. The paper consolidates the concepts leading to a family of generalised nonhydrostatic NFTFV flow solvers that include soundproof PDEs of incompressible Boussinesq, anelastic and pseudoincompressible systems, common in large-eddy simulation of small- and meso-scale dynamics, as well as all-scale compressible Euler equations. Such a framework naturally extends predictive skills of large-eddy simulation to the global atmosphere, providing a bottom-up alternative to the reverse approach pursued in the weatherprediction models. Theoretical considerations are substantiated by calculations attesting to the versatility and efficacy of the NFTFV approach. Some prospective developments are also discussed.
\end{abstract}

Key words: unstructured mesh atmospheric models, nonoscillatory forward-in-time schemes, anelastic equations, compressible low Mach number flows, atmospheric flows

PACS:

Preprint submitted to J. Comput. Phys.

28 June 2016 


\section{Introduction}

\subsection{Historical background}

Weather forecasts have been one of the focal areas of numerical modelling since the advent of electronic computers $[16,2,86]$ and the creation of the JCP $[4,176]$. From the basic ideas of numerical weather and climate modelling developed a century ago, the computational meteorology went a long way to become one of the prominent disciplines at the frontiers of computational physics $[97,1,9]$. Advances in computational meteorology are primarily driven by weather forecasting and its verification, by means of data assimilation, against continuous flow of observations. However, meteorology is a broad discipline with multiple research areas and foci of knowledge. Their traditional hierarchy of alignment - often reflected by the names of divisions at atmospheric research centres - corresponds to the spatio/temporal scales of studied phenomena, from micro-scale of hydrometeor particulates, through small- and meso-scales of cloud evolution and flows over complex terrain, through synoptic weather phenomena, to the planetary scale of global weather and climate. All such areas rely on PDEs of mathematical physics, the solution of which necessitates the use of numerical methods. As the properties of atmospheric motions vary across scales [26,62], the corresponding areas of computational meteorology develop domain specific expertise and ultimately contribute diverse approaches to the advancement of weather forecasts and climate models.

The literature devoted to numerical methods underlying advancements of weather and climate models is vast. According to the Thomson Reuters Web of Science, over 1300 papers has been published since 2000 on the topic "numerical models for weather and climate", with the cumulative citation count of $\approx 30,000$. Consequently, there is already available a substantial number of related reviews, developed from various angles and through varied methodological preferences, many of which are referenced in this paper. In particular, we refer the interested reader to $[26,62]$ for indepth discussions of theories guiding multiscale atmospheric modelling and to [146,177,88,144,23,95], among the others, for specialised reviews of the numerical methods explored in dynamical cores of atmospheric models. To avoid duplicating the existing literature, we instead outline the historical background to the interdisciplinary crossfertilisation at the foundation of the all-scale atmospheric models, with particular emphasis on a select class of versatile finite-volume methods discussed in the body of the paper. The order of the presentation reflects the bottom-up approach of our quest for simulating all-scale atmospheric dynamics.

\footnotetext{
* Corresponding Author.

Email address: smolar@ecmwf.int (Piotr K. Smolarkiewicz).
} 
Historically, atmospheric models across scales were dominated by finite-difference (FD) and spectral-transform methods for spatial discretisation of their governing PDEs [177]. In particular, finite-difference methods operating on regular rectangular grids have prevailed in small and mesoscale models for research of cloud processes and orographic flows [64,123], with terrain fitted grids mimicked by continuous mappings $[40,20,171]$ and horizontal resolution refinement delegated to nested grids [21]. These techniques are still prevailing in computational studies from planetary boundary layer to regional climate $[145,38]$, which is not surprising given the sophistication of finite-differencing used in atmospheric models already in the nineteen sixties. The work [4], in the inaugural issue of JCP, introduced higher-order centred (viz. non-dissipative) FD schemes conserving mean kinetic energy and enstrophy, thus preventing notorious nonlinear instability marring the early weather codes [82]. This had a major impact on the field by: a) enabling long-term simulations of weather and climate; b) engendering a variety of FD schemes enhancing desired aspects of accuracy in atmospheric models [157]; and c) redirecting the action of artificial (nonlinear in shear) viscosity [103] from stabilising/regularising the solution to parametrising subgrid-scale eddy motions $[81,120]$ — while laying foundations for large-eddy simulation [77]. Notably, conserving discrete analogues of mean quadratic quantities is distinct from simulating hyperbolic conservation laws of gas dynamics $[45,75]$, where the use of locally conservative numerical methods is the first prerequisite for accurate representation of shock waves in supersonic flows. The atmospheric motions are generally characterised by low Mach numbers, and subject to at most weak acoustic discontinuities [134]. Consequently, their numerical simulation may favour distinct conservation principles [157]. Notwithstanding the disparity of applications, small- to meso-scale FD models in the nineteen seventies drew inspiration from advances in computational aerodynamic and mechanical engineering. A substantial example is a nonhydrostatic anelastic model in terrain-following coordinates [20] that adopted the strong conservation formulation $[73,3,165]$ together with the pressure projection [19] to a centred (in time and space; the latter staggered after [50]) discretisation of the governing equations, while using an advanced direct solver for the resulting discrete Poisson problem. This accomplished conservation of mass, momentum and entropy fluctuations, while controlling the global quadratic invariant and enabling large-eddy simulations (LES) in a curvilinear coordinate frame.

Armed with non-dissipative spectral transform and finite-difference methods, the atmospheric community renounced upwind differencing schemes, as overly dissipative albeit nonlinearly stable [82], ${ }^{1}$ essentially in all areas of atmospheric simulation, but a few holdouts including cloud physics. Simulation of

\footnotetext{
${ }^{1}$ Lilly implies the Lelevier's type first-order upwinding [111] rather than upwind differencing per se, as ibidem he also acknowledges "high-accuracy monotone upwind schemes" and "semi-Lagrangian schemes".
} 
clouds must account for phase changes of water substance, the mathematical description of which does not tolerate negative amounts of water substance generated by dispersive oscillations characteristic of higher-order linear advection schemes. Despite the efforts in minimising dispersion of advection schemes [39], the first-order-accurate flux-form upwind was one of a few viable options available for transport of water species in early cloud models [140]. In this context, the Godunov theorem [45] - revealing that linear schemes guaranteeing smooth solutions and the overall accuracy of second- and higher-order methods do not exist - become most consequential for cross-fertilising all areas of computational fluid dynamics (CFD). In essence, it left no alternative but to either accept first-order methods with their notorious implicit diffusivity, or to abandon the premise of linearity, and complicate the problem by approximating even the simplest constant-coefficient advection equation with an elaborate nonlinear scheme. The second choice gave rise to nonoscillatory flux-form methods for integrating the conservation laws, pioneered with such renown approaches as flux-corrected transport (FCT) [12,189], monotonic upstream-centred scheme for conservation laws (MUSCL) [163,164], high resolution schemes for hyperbolic conservation laws (TVD) [53], and the piecewise parabolic method (PPM) [22] - all quickly embraced by computational meteorology, primarily for conservative tracer transport in cloud and chemistry models [113]. The interdisciplinary importance of the nonoscillatory methods has been amplified in the nineteen nineties, as successful simulations of turbulent flows reported in diverse research areas used nonoscillatory advection schemes to model subgrid effects $[105,108,83,91]$ - establishing the legitimacy of implicit large-eddy simulation (ILES), widely reviewed in [48] a decade later.

Regardless of all these spectacular advancements of the FD schemes, the rigid connectivity of their underlying, topologically rectangular grids has been long recognised [112] as an obstacle for effective flow simulation in complex geometric configurations that cannot be efficiently accommodated with global mappings. ${ }^{2}$ The nonoscillatory finite-volume (FV) methods on unstructured meshes, advanced in the nineteen eighties $[58,78,8]$, extended functionality of flux-form finite differencing to arbitrary arrangements of nodes (where discrete solutions are sought) and definitely distinguished FV from FD. ${ }^{3}$ Because the mathematical formalism underlying $\mathrm{FV}$ methods is that of the vector differential calculus - or, more generally, integration of differential forms - the FV approach naturally lends itself to discrete integrations of conservation laws on manifolds. For example, a volume integral of the divergence of a vector

\footnotetext{
2 According to the Thomson Reuters Web of Science, [112] was the first article in a learned journal that used the "finite volume" term in the context of numerical methods; similarly, [78] was the first in the JCP literature.

3 In some publications, flux-form FD schemes can be labelled as FV and attributed to Godunov [45]—who himself refers to FD [46].
} 
flux field over an arbitrary shaped computational cell is evaluated as a surface integral of a flux through the cell boundaries. Consequently, reconstruction of surface fluxes from cell mean values of the fluxed variables becomes a key element of the FV approach. Importantly, it can draw from the experience with nonoscillatory flux-form finite differencing, without substantial complications for spatial discretisation up to the second order of accuracy. Because the flux reconstruction does not require the sampled data to be aligned, higher order formulations are in principle possible and are the subject of active research $[183,184]$. Due to these intrinsic strengths, FV is the first choice approach for general purpose, commercial and open-source CFD software.

In computational meteorology, the FV philosophy gained popularity for integrating conservative Lagrangian tracer transport in chemistry models, as extensively reviewed in a dedicated chapter [88]. In particular, it spawned a bespoke class of fully conservative, optionally nonoscillatory, semi-Lagrangian schemes $[179,180,100,101,71,72]$ that extend standard point-wise semi-Lagrangian advection $[142,126]$ to trajectory-wise advection of finite volumes, while circumventing the CFL stability condition characteristic of the Eulerian schemes. The approach also has been adapted for integrating the mass continuity equation in weather and climate models [143], but found limited application in operations due to its computational expense [190]. In the area of all scale (viz. nonhydrostatic) atmospheric dynamics of primary interest to this paper, a trend towards FV methods is still emerging - cf. [114,162,119] for technical expositions and $[23,95]$ for recent reviews - even though the existing FV implementations still lag behind the established spectral-transform codes in terms of the time-to-solution and energy efficiency [172].

Like with finite-difference methods, there is a variety of finite-volume schemes and discretisation stencils. In areas of numerical modelling accustomed to regular finite-difference stencils, grids are often dubbed "unstructured" whenever they depart from standard Cartesian arrangement in the computational space [144]. In this paper we restrict attention to simulations using flexible unstructured meshes in the sense of engineering CFD; where a mesh, consisting of either irregular or regular elements, is referred to as unstructured if the discretisation associated with it does not rely on a systematic principle for identification of neighbouring points - the examples of which include flexible element and edge-based connectivity, in contrast to structured data arrangements such as i, j, k indexing or binary trees $[170,156]$. The corollary is that even a structured grid qualifies as an unstructured mesh when used in a numerical model that does not depend on the particular structure of such a grid.

Meteorological interest in unstructured meshing dates back to the nineteen sixties in the context of uniform horizontal discretisation for global flows, sti- 
fled a decade later by the success of spectral transforms [177]. ${ }^{4}$ The intrinsic powers of the FV approach have not been put to practical use until the beginning of the current century, in the framework of the OMEGA model [5] for forecasting high-impact weather, air quality, and environmental hazard. Since then, there has been a continuously growing interest in modelling atmospheric flows on unstructured meshes and especially in utilising flexible mesh adaptivity $[104,55]$. Because unstructured-meshes are essentially free of topological constraints, they offer flexibility unmatched by the established techniques operating on regular Cartesian grids. Admittedly, such grids enable computationally efficient static and dynamic mesh adaptivity via continuous mappings $[109,171,66,13,14]$, yet their rigid connectivity imposes stringent constraints on the adapted grids. The unavailability of regular equidistant discretisation on a spherical surface is an apparent evidence of these constraints and a venerable force for advancement of flexible meshing in atmospheric models. However, benefits of the flexible meshing are not limited to global flows, and they can be equally important at finer-scale problems of computational meteorology; for example, in research and forecasting of weather in long winding valleys and mountainous areas, or onset and evolution of extreme events.

Notwithstanding potential benefits of modelling atmospheric flows with unstructured meshes and growing interest in their use, the unstructured-mesh atmospheric models are still relatively new and have not yet achieved the recognition of structured-grid models commonly used in research and operations. An important specificity of the atmospheric dynamics is that it constitutes a relatively small perturbation about dominant balances of hydrostacy, geostrophy and thermal winds established in effect of the Earth gravity, rotation, stablystratified thermal structure of its atmosphere and the incoming flux of solar energy. ${ }^{5}$ Preserving this fundamental equilibrium, while accurately resolving the perturbations about it, conditions the design of atmospheric models and subjects their numerical procedures to intricate stability and accuracy requirements. This specificity of atmospheric flows poses new challenges to anisotropic heterogeneous discretisation and flexible mesh adaptivity [32], largely developed in the engineering community for neutrally stratified non-rotating flows throughout a range of Mach number regimes $[178,98]$. Compared to engineering flows, gravity and planetary rotation provide for restoring forces generat-

${ }^{4}$ In the nineteen seventies the spherical harmonics method became the method of choice for global simulations, and combined over a period with two-time-level semiimplicit semi-Lagrangian time stepping it became a marvel of numerical weather prediction at hydrostatic resolutions (NWP) $[177,172]$. However, the progress of distributed computing in the nineteen nineties revealed efficiency limits of the spherical harmonics, and reinvigorated research into compact-stencil discretisations including the unstructured meshing.

${ }^{5}$ Consider that the total conversion of available potential energy to kinetic energy is only $0.26 \%$ of the incoming solar radiation at the top of the atmosphere [172]. 
ing continuous spectra of intricate dispersive wave phenomena, energetically overwhelming hyperbolic (sound) wave dynamics. The associated Froude and Rossby numbers, on top of the low Mach and high Reynolds numbers, give rise to multiplicity of scaling regimes underlying weather and climate [62]. Furthermore, scale-specific processes that are the response to mechanical and thermal forcing and are not primarily the result of dynamical flow instabilities, ${ }^{6}$ add another dimension to the scaling regimes [90]. While each regime could be described with its own model equations, the efficacy of operational forecasting, research and education gives priority to unified theoretical/numerical models that cover multiple spatio-temporal scales of atmospheric dynamics.

The quest for unified CFD models can be traced back to implicit continuousfluid Eulerian (ICE) method [51,52] applicable to all speed flows, from incompressible to hypersonic regime, in multidimensional domains. Basically, ICE extends the marker-in-the-cell (MAC) approach [50] for viscous incompressible flows to an implicit finite-difference solver of full nonlinear equations of gas dynamics. Interestingly, it depends on the solution of a linear elliptic Helmholtz problem for a density perturbation, that in the incompressible limit becomes effectively a Poisson problem for pressure reminiscent of the projection approaches [50,19]. Over the years many ingenious methods have been devised to extend concepts underlying compressible flow solvers to low-Mach number regime $[87,61,49,161,34,166,174,116]$ and/or generalise incompressible concepts to compressible flows $[57,60,191,185,11,186,99,181,182]$. In computational meteorology the progress of unified models was motivated by the specificity of atmospheric dynamics and goals of weather and climate prediction. The advance of massively parallel computing in the nineteen nineties has stimulated the development of nonhydrostatic models. With decreasing grid intervals, operational NWP codes naturally evolved towards extending their proven hydrostatic apparatus to the fully compressible Euler equations $[187,28,74,10,117,6,173]$; whereas cloud-scale and mesoscale nonhydrostatic research models originated in the nineteen seventies $[153,20,64]$ were extending their functionality by increasing the spatial domain [129,114,118,119,44]. At present, there is already substantial experience and the accumulated literature on integrating the all-scale Euler equations for weather and climate; see $[146,23,95]$ for reviews; however, no current NWP model runs globally at nonhydrostatic resolutions in operations. Such high resolutions are still computationally unaffordable and too inefficient to meet demands of the limited time window for distributing global forecasts to the end users. The recent work [139] shows how the ability of simulating global all-scale atmospheric dynamics on unstructured meshes can complement established NWP models and aid the progress - this motivates the technical content of this paper.

\footnotetext{
$\overline{6}$ Such processes are associated with the distribution of solar incoming radiation, topography, continents and oceans, vegetation, soil and other land-use.
} 


\subsection{Technical scope}

This paper consolidates a decade of systematic efforts on generalising proven nonoscillatory forward-in-time semi-implicit flux-form finite-difference (NFTFD) integrators of all-scale atmospheric PDEs to unstructured meshes and FV discretisations. One defining aspect of the NFTFD schemes is a forward-in-time (FT) two-time-level discretisation of the governing conservation laws. In order to achieve an at least second-order accuracy in time, derivations of FT schemes convert temporal derivatives to spatial derivatives by exploiting the analytic dependencies of temporal and spatial partial derivatives in the governing PDEs - a general Lax-Wendroff concept [75], recently also referred to as the Cauchy-Kowalevski procedure [159]. The FT integrators have a long history not only in CFD, but also in computational meteorology, especially in the context of conservative advection $[76,25,122,160]$. The acknowledged advantages of the FT advection schemes are the reduced storage compared to multi-time-level schemes and the absence of computational modes characteristic of centred-in-time methods. Owing to the generality of the CauchyKowalevski procedure, the FT schemes are easily adaptable to specific needs of governing theoretical models, admitting arbitrary dimensionality $[33,122]$, geometry $[127,109]$ and physics $[127,128,47,136]$.

The other defining aspect of the NFTFD schemes is their reliance on the nonoscillatory advection algorithms. Over decades many nonoscillatory schemes were developed, forthcoming from different physical insights inherent in their composition [48]. One such scheme, known as the multidimensional positive definite advection transport algorithm (MPDATA) [124], has been devised specifically for atmospheric cloud simulations, where positivity of water vapour and cloud condensate is imperative. For this reason MPDATA originated as an iterated upwind scheme exploiting the sign-preserving property of upstream differencing. Over decades, MPDATA evolved into a family of solvers for systems of generalised transport equations [59] targeting conservation laws of atmospheric dynamics. Besides standard sign-preservation and nonlinear stability at the second-order accuracy for arbitrary flows, MPDATA also provides implicit turbulence modelling capability to the full set of equations. The implicit large eddy simulation (ILES) properties of MPDATA-based high-Reynolds-number solvers were widely documented in the context of structured grids $[92,93,30,94,167]$ and played a key role in extending the realm of the FT integrators to general fluid models [110], proven for a range of computational studies in all scale atmospheric dynamics $[138,68-70]$ and beyond $[24,169,41,54,67]$.

The potential of MPDATA for unstructured mesh modelling was recognised already in the nineteen nineties, first in context of mantle convection models [15], and then in the area of weather and environmental modelling [5]. 
However its ab initio derivation $[130,131]$ was the first to encompass error compensating terms required for unstructured meshes and laid the ground for the MPDATA based NFTFV all-scale atmospheric flow solvers. Initial developments were first verified in the context of engineering gas dynamics for all speed flows $[133,134]$ using a selection of techniques for mesh adaptivity [178,147-149]. These works documented solution accuracy and convergence and demonstrated favourable comparisons to established benchmarks (theoretical and numerical). Subsequent developments addressed meteorological applications producing models for simulating idealised hydrostatic dynamics of the planetary atmosphere [150], reduced 2D soundproof models for simulation of nonhydrostatic gravity-wave dynamics [151,135] and their consequent generalisation to 3D mesoscale modelling of nonhydrostatic dynamics $[137,152]$ - all supported with extensive verification studies using variety of meshes with different degree of anisotropy and heterogeneity, in two and three spatial dimensions. Verified in simulations with intricate stratified rotating flows the approach performs well for arbitrary shaped meshes and matches the accuracy of the NFTFD solvers on Cartesian grids.

The next section outlines the system of generalised nonhydrostatic PDEs including soundproof equations common in research of small-to-mesoscale dynamics and the fully compressible Euler equations favoured in high-resolution simulation of global dynamics. For simplicity, the presentation is restricted to dry motions and idealised heat sinks/sources and momentum dissipation. Applications of the generalised system with account of moist processes and more realistic scenarios can be found in $[69,70]$, whereas extension of the NFTFV solvers to moist precipitating dynamics will be reported in a separate study. Section 3 summarizes the numerics of the NFTFV solvers, starting with the elemental FT template algorithm that forms the building block for the subsequently discussed elaborate semi-implicit integrators of the generalised nonhydrostatic PDEs. The theoretical considerations of section 3 come to life in section 4 , where the versatility and efficacy of the approach are demonstrated with flow problems across a range of scales and applications, from convective boundary layer, to gravity wave dynamics and orographic flows, to global circulation epitomising planetary weather. Section 5 highlights current research on development of atmospheric general circulation models and concludes the paper.

\section{ANALYTIC FORMULATION}

The generalised all-scale system of PDEs can be compactly written as

$$
\frac{\partial \mathcal{G} \varrho}{\partial t}+\nabla \cdot(\mathcal{G} \varrho \mathbf{v})=0
$$




$$
\begin{aligned}
& \frac{\partial \mathcal{G} \varrho \theta^{\prime}}{\partial t}+\nabla \cdot\left(\mathcal{G} \varrho \mathbf{v} \theta^{\prime}\right)=-\mathcal{G} \varrho\left(\widetilde{\mathbf{G}}^{T} \mathbf{u} \cdot \nabla \theta_{a}-\mathcal{H}\right) \\
& \frac{\partial \mathcal{G} \varrho \mathbf{u}}{\partial t}+\nabla \cdot(\mathcal{G} \varrho \mathbf{v} \otimes \mathbf{u})= \\
& -\mathcal{G} \varrho\left(\Theta \widetilde{\mathbf{G}} \nabla \varphi+\mathbf{g} \Upsilon_{B} \frac{\theta^{\prime}}{\theta_{b}}+\mathbf{f} \times\left(\mathbf{u}-\Upsilon_{C} \mathbf{u}_{a}\right)-\mathcal{M}^{\prime}\left(\mathbf{u}, \mathbf{u}_{a}, \Upsilon_{C}\right)-\boldsymbol{D}\right)
\end{aligned}
$$

The system (1) encloses three distinct sets of the governing equations: the fully compressible Euler equations under gravity in a rotating reference frame; and their two reduced soundproof forms ${ }^{7}$ the pseudo-incompressible equations of Durran [35] and the anelastic equations of Lipps-Hemler [84,85]. The latter set includes incompressible Boussinesq equations [141] as a special case. The distinction between the different sets is encrypted in definitions, collected in table 1 , of the generalised density $\varrho$ and pressure variable $\varphi$, together with the corresponding dimensionless coefficients $\Theta, \Upsilon_{B}$, and $\Upsilon_{C}$ that depend on various states of the potential temperature $\theta$. For ideal gas, $\theta$ amounts to specific entropy via $d s=c_{p} d \ln \theta$, with $c_{p}$ denoting the specific heat at constant pressure. Hereafter, $\rho$ denotes the air density, while subscripts $b, 0$ and $a$ mark, respectively, a horizontally homogeneous and hydrostatically balanced base state, a constant reference value and the ambient state discussed later in this section. The generalised pressure $\varphi$ depends on the Exner pressure $\pi \equiv$ $\left(p / p_{0}\right)^{R_{d} / c_{p}}$, where $R_{d}$ is the gas constant for dry air. Unless stated otherwise, the primes denote perturbations with respect to the ambient state; e.g., $\pi^{\prime}=$ $\pi-\pi_{a}, \theta^{\prime}=\theta-\theta_{a}$, and so forth.

\begin{tabular}{|l|l|l|l|}
\hline & compressible & pseudo-incompressible & anelastic \\
\hline$\varrho$ & $\rho(\mathbf{x}, t)$ & $\rho_{b}(z) \theta_{b}(z) / \theta(\mathbf{x}, t)$ & $\rho_{b}(z)$ \\
\hline$\varphi$ & $c_{p} \theta_{0} \pi^{\prime}$ & $c_{p} \theta_{0} \pi^{\prime}$ & $c_{p} \theta_{b}(z) \pi^{\prime}$ \\
\hline$\Theta$ & $\theta(\mathbf{x}, t) / \theta_{0}$ & $\theta(\mathbf{x}, t) / \theta_{0}$ & 1 \\
\hline$\Upsilon_{B}$ & $\theta_{b}(z) / \theta_{a}(\mathbf{x})$ & $\theta_{b}(z) / \theta_{a}(\mathbf{x})$ & 1 \\
\hline$\Upsilon_{C}$ & $\theta(\mathbf{x}, t) / \theta_{a}(\mathbf{x})$ & $\theta(\mathbf{x}, t) / \theta_{a}(\mathbf{x})$ & 1 \\
\hline
\end{tabular}

Table 1

Definitions of the density/pressure variables and coefficients $\Theta, \Upsilon_{B}$, and $\Upsilon_{C}$ in (1)

Notably, the soundproof equations contained in (1) do not require the provision of constitutive laws for their solution, because their respective pressure

\footnotetext{
$\overline{7}$ In contrast to the soundproof systems, the Euler equations are free of any linearisations that discard perturbational terms as negligibly small; see $[27,63,138]$ for extended discussions of validity regimes of soundproof PDEs.
} 
perturbations are determined from the elliptic equations that follow from constraining the velocity solutions to satisfy mass continuity. In other words, their constitutive laws were analytically accounted for while deriving the reduced equations, and afterwards are not required for the solution. Generally, this is not the case with fully compressible equations where the ideal gas law

$$
\varphi=c_{p} \theta_{0}\left[\left(\frac{R_{d}}{p_{0}} \varrho \theta\right)^{R_{d} / c_{v}}-\pi_{a}\right] .
$$

explicitly relates the thermodynamic pressure perturbations to the distribution of temperature and mass in the fluid.

For the three sets of PDEs included in (1), the mathematical symbolism anticipates the strong conservation formulation $[165,20]$ and already accounts for the generalised time-dependent curvilinear coordinates enabling the representation of orography or dynamic mesh adaptivity $[109,171,66]$. In particular, $(\mathbf{x}, t)$ refers to the coordinates of the generalised time-dependent frame, and $\mathcal{G}(\mathbf{x}, t)$ denotes the Jacobian-so, $\mathcal{G}^{2}$ is the determinant of the metric tensor that defines the fundamental metric in a space of interest where the problem is solved [109]. Furthermore, $\nabla \cdot(.$.$) denotes the scalar product of spatial partial$ derivatives with a vector, so the total derivative underlying conservation form $(1), d / d t=\partial / \partial t+\mathbf{v} \cdot \nabla$, takes the velocity $\mathbf{v}=\dot{\mathbf{x}}$ not necessarily equal to the physical velocity $\mathbf{u}$ for which equations are solved. The $\widetilde{\mathbf{G}} \nabla \varphi$ in the momentum equation symbolises the product of a known matrix of metric coefficients and the vector of partial derivatives, whereas $\widetilde{\mathbf{G}}^{T} \mathbf{u}=\mathbf{v}-\mathbf{v}^{g}$ on the rhs of the entropy equation accounts for the mesh velocity $\mathbf{v}^{g}$, set to zero in the remainder of this paper. For the reader's convenience, various metric coefficients are exemplified for the spherical frame in Appendix.

The remaining symbols are specific to applications addressed in the paper. In the entropy equation $(1 \mathrm{~b}), \mathcal{H}$ symbolises a heat source/sink. In the momentum equation (1c), the Coriolis parameter is given as $\mathbf{f} \equiv 2 \boldsymbol{\Omega}$, where $\boldsymbol{\Omega}$ denotes a constant angular velocity of the rotating reference frame. The gravitational acceleration $\mathbf{g}=(0,0,-g)$ enters the buoyancy term on the rhs of (1c). The term $\mathcal{M}^{\prime}\left(\mathbf{u}, \mathbf{u}_{a}, \Upsilon_{C}\right)=\mathcal{M}(\mathbf{u})-\Upsilon_{C} \mathcal{M}\left(\mathbf{u}_{a}\right)$ symbolises metric forcings (both specified in Appendix for the spherical domain), whereas $\boldsymbol{D}$ denotes a momentum sink.

The generalised system of PDEs (1) exploits an auxiliary ambient state $\left(\mathbf{u}_{a}, \phi_{a}, \theta_{a}\right)$ assumed to be a known particular solution of the governing PDEs, typically different for each set of PDEs contained in (1) [138]. The primary role of ambient states is to simplify the design of the initial and boundary conditions as well as to enhance the accuracy of calculations in finite-precision arithmetic. Generally, ambient states can be time-dependent; e.g., prescribing oceanic tidal motions [169]. In this paper, only stationary ambient states are 
considered, e.g., geostrophically-balanced thermal wind for momentum equations, together with identically satisfied ambient mass continuity and entropy equations. Because such an ambient state derives as a compatibility condition of the governing PDE system, it does not impose any approximations on the governing equations but conveniently defines the perturbation variables [138].

\section{NUMERICAL APPROXIMATIONS}

\subsection{Forward-in-time template algorithm}

To design computer programs for integrating (1), it is convenient to view all individual PDEs in (1) as a generalised transport equation for an arbitrary scalar variable $\Psi$,

$$
\frac{\partial G \Psi}{\partial t}+\nabla \cdot(\mathbf{V} \Psi)=G \mathcal{R}
$$

where vector field V (hereafter, "advector") as well as scalar fields $G$ and $\mathcal{R}$ are assumed to be known functions of time and space. Depending on the definitions of $G, \Psi, \mathbf{V}$ and $\mathcal{R},(3)$ expresses either (1a), (1b) or (1c), as specified in table 2; see [138] for a comprehensive discussion. For anelastic PDEs, the density $\varrho$ is prescribed (cf. table 1), and (1a) reduces to a diagnostic constraint, eventually leading to the elliptic boundary value problem for pressure perturbation $\varphi$. For either soundproof or compressible PDEs the cumulative mass flux, $\mathbf{V} \Psi=\mathcal{G} \mathbf{v} \varrho$ in the mass continuity equation, amounts to transportive momenta in the entropy and the momentum equations. The latter assures the compatibility of conservative advection of $\theta^{\prime}$ and components of $\mathbf{u}$ with the mass continuity, preserving consistency of the entropy and momentum conservation laws with their Lagrangian form $\dot{\Psi}=\mathcal{R}$.

\begin{tabular}{|l|l|l|l|l|}
\hline equation & $\Psi$ & $\mathbf{V}$ & $G$ & $\mathcal{R}$ \\
\hline$(1 \mathrm{a})$ & $\varrho$ & $\mathcal{G} \mathbf{v}$ & $\mathcal{G}$ & 0 \\
\hline$(1 \mathrm{~b})$ & $\theta^{\prime}$ & $\mathcal{G} \varrho \mathbf{v}$ & $\mathcal{G} \varrho$ & $\operatorname{rhs}(1 \mathrm{~b})$ \\
\hline$(1 \mathrm{c})$ & $\mathbf{u}$ & $\mathcal{G} \varrho \mathbf{v}$ & $\mathcal{G} \varrho$ & $\operatorname{rhs}(1 \mathrm{c})$ \\
\hline
\end{tabular}

Table 2

Definitions of $\Psi, \mathbf{V}$ and $G$ in (3) for corresponding PDEs of the system (1)

A minimal common element of our all NFTFV schemes for integrating (1) is a second-order-accurate FT template for (3)

$$
\Psi_{\mathbf{i}}^{n+1}=\mathcal{A}_{\mathbf{i}}\left(\widetilde{\Psi}^{n}, \mathbf{V}^{n+1 / 2}, G^{n}, G^{n+1}\right)+0.5 \delta t \mathcal{R}_{\mathbf{i}}^{n+1}, \quad \widetilde{\Psi}^{n} \equiv \Psi^{n}+0.5 \delta t \mathcal{R}^{n},
$$


where $\mathcal{A}$ is a shorthand for the MPDATA transport operator MPDATA [138]. Furthermore, the index $\mathbf{i}$ symbolises position on the computational grid, $\delta t$ is a time interval between two consecutive time levels denoted by $n$ and $n+1$, and the "advector" $\mathbf{V}^{n+1 / 2}$ is an $\mathcal{O}\left(\delta t^{2}\right)$ estimate of $\mathbf{V}$ at the intermediate time level. The template (4) derives from the modified equation analysis [168] of the forward-in-time differencing for (3), while using the Cauchy-Kovaleski procedure to determine a means of compensating the $\mathcal{O}(\delta t)$ error-terms (of the modified equation) with spatial differencing $[127,131,132]$.

\subsection{Semi-implicit integrators}

In the system (1) only the mass continuity equation (1a) is homogeneous, whereas the entropy and momentum equations have non-vanishing right-handsides that generally depend on all model variables. In the two-time-level framework, the latter favours implicit representations of the rhs forcings, not only for the stability but also the overall accuracy of the solutions $[65,31]$. Thanks to the homogeneity of (1a), the entire model algorithm for (1) can be naturally reduced to the two distinct steps.

The first step provides the advectors for the first term on the rhs of (4). For anelastic or adiabatic pseudo-incompressible PDEs illustrated in this paper, $\mathbf{V}^{n+1 / 2}=(\mathcal{G} \varrho \mathbf{v})^{n+1 / 2}$ is evaluated by linear extrapolation from $t^{n-1}$ and $t^{n}$ and used consistently for all prognostic variables. For compressible PDEs the linearly extrapolated $\mathbf{V}^{n+1 / 2}=(\mathcal{G} \mathbf{v})^{n+1 / 2}$ is used only in the density advection,

$$
\varrho_{\mathbf{i}}^{n+1}=\mathcal{A}_{\mathbf{i}}\left(\varrho^{n},(\mathcal{G} \mathbf{v})^{n+1 / 2}, \mathcal{G}^{n}, \mathcal{G}^{n+1}\right) \quad \Longrightarrow \quad \mathbf{V}^{n+1 / 2}=\overline{(\mathcal{G} \varrho \mathbf{v}}^{n+1 / 2}
$$

while updating the density and concomitantly evaluating the advectors $\mathbf{V}^{n+1 / 2}$ as cumulative directional mass fluxes for advection of $\theta^{\prime}$ and all components of $\mathbf{u}$; see [138] for an exposition.

The second step integrates the entropy and momentum equations in (1). To account for the nonlinearity of the rhs-here, due to the coefficients $\Theta, \Upsilon_{C}$ and the metric terms $\mathcal{M}$ - the template algorithm (4) is, generally, executed iteratively lagging nonlinear terms behind. ${ }^{8}$ To simplify notation, the lagged terms will be denoted by the superscript ${ }^{*}$, whereas variables without superscripts will represent subsequent iterates of the solution future values at $t^{n+1}$. Consequently,

\footnotetext{
8 A standard fixed-point iteration rooted in the Banach Principle - theorem VIII.1.1 in [96] — is quite effective when applied judiciously; see [134,136,138] for implementations in, respectively, gas dynamics, magnetohydrodynamics and generalised PDEs (1), all in the context of the FT template (4).
} 


$$
\begin{aligned}
\theta_{\mathbf{i}}^{\prime}=\widehat{\theta}_{\mathbf{i}}^{\prime}- & 0.5 \delta t\left(\widetilde{\mathbf{G}}^{T} \mathbf{u} \cdot \nabla \theta_{a}\right)_{\mathbf{i}} \\
\mathbf{u}_{\mathbf{i}}=\widehat{\mathbf{u}}_{\mathbf{i}}- & 0.5 \delta t\left(\Theta^{\star} \widetilde{\mathbf{G}} \nabla \varphi+\mathbf{g} \Upsilon_{B} \frac{\theta^{\prime}}{\theta_{b}}\right)_{\mathbf{i}} \\
& \quad-0.5 \delta t\left(\mathbf{f} \times\left(\mathbf{u}-\Upsilon_{C}^{\star} \mathbf{u}_{a}\right)-\mathcal{M}^{\prime}\left(\mathbf{u}^{\star}, \mathbf{u}_{a}, \Upsilon_{C}^{\star}\right)_{\mathbf{i}} .\right.
\end{aligned}
$$

Here, $\widehat{\theta}_{\mathbf{i}}^{\prime}$ and $\widehat{\mathbf{u}}_{\mathbf{i}}$ are the shorthands for the transport operator $\mathcal{A}$ applied to $\widetilde{\theta}^{\prime}$ and $\widetilde{\mathbf{u}}$ in (4), subsumed with the respective contributions from forcings $\mathcal{H}$ and $\mathcal{D}$ estimated to $\mathcal{O}(\delta t)$ at $t^{n+1}$, given as

$$
\begin{aligned}
& \widehat{\theta}_{\mathbf{i}}^{\prime}=\mathcal{A}_{\mathbf{i}}\left(\widetilde{\theta}^{\prime}+0.5 \delta t \mathcal{H}^{n+1}, \mathbf{V}^{n+1 / 2}, \varrho^{* n}, \varrho^{* n+1}\right) \\
& \widehat{\mathbf{u}}_{\mathbf{i}}=\mathcal{A}_{\mathbf{i}}\left(\widetilde{\mathbf{u}}+0.5 \delta t \mathcal{D}^{n+1}, \mathbf{V}^{n+1 / 2}, \varrho^{* n}, \varrho^{* n+1}\right)
\end{aligned}
$$

with $\mathbf{V}^{n+1 / 2}$ provided by the preceding step, and the effective densities $\varrho^{* n}$ and $\varrho^{* n+1}$ defined, respectively, as $\varrho^{*^{n}}:=\mathcal{G}^{n} \varrho^{n}$ and $\varrho^{* n+1}:=\mathcal{G}^{n+1} \varrho^{n+1}$. Furthermore,

$$
\theta_{\mathbf{i}}=\left(\widehat{\theta}^{\prime}-0.5 \delta t \widetilde{\mathbf{G}}^{T} \mathbf{u} \cdot \nabla \theta_{a}+\theta_{a}\right)_{\mathbf{i}}
$$

the first guess of which is generated by advecting $\theta$ as

$$
\theta_{\mathbf{i}}^{\star}=\mathcal{A}_{\mathbf{i}}\left(\theta^{n}+\delta t \mathcal{H}^{n}, \mathbf{V}^{n+1 / 2}, \varrho^{* n}, \varrho^{* n+1}\right)
$$

The first guess $\mathbf{u}_{\mathbf{i}}^{\star}$ is obtained by linear extrapolation from $t^{n-1}$ and $t^{n}$ to $t^{n+1}$. With this design, the solution is fully second order accurate even for a single iteration, and two iterations give already close approximation to the trapezoidal integral [134].

The scheme outlined in (6)-(8) contains implicit trapezoidal integrals of pressure gradient, buoyancy and Coriolis terms, with coefficients depending on full potential temperature and metric forces evaluated explicitly. ${ }^{9}$ Regardless of the selected option, derivation of the closed-form expression for the velocity update is common to all three sets of PDEs. The future value of $\theta^{\prime}$ is substituted in the buoyancy term of the momentum equation with the rhs of the entropy scheme, and all terms depending on the future value of $\mathbf{u}$ are gathered on the lhs of the momentum scheme - while dropping the spatial grid index $\mathbf{i}$ everywhere, as all dependent variables, coefficients and terms are colocated in (6)-(8). This results in

9 Optionally, the compressible pressure can be evaluated directly from the gas law (2), leading to the acoustic option of the solver in the spirit of gas dynamics for high speed flows [134] - a useful reference for large time step simulations of low Mach number flows $[138,69]$. 


$$
\begin{aligned}
\mathbf{u}+0.5 \delta t \mathbf{f} \times \mathbf{u}-(0.5 \delta t)^{2} \mathbf{g} \Upsilon_{B} \frac{1}{\theta_{b}} \widetilde{\mathbf{G}}^{T} \mathbf{u} \cdot \nabla \theta_{a}= \\
\widehat{\mathbf{u}}-0.5 \delta t\left(\mathbf{g} \Upsilon_{B} \frac{\widehat{\theta}^{\prime}}{\theta_{b}}-\mathbf{f} \times \Upsilon_{C}^{\star} \mathbf{u}_{a}-\mathcal{M}^{\prime}\left(\mathbf{u}^{\star}, \mathbf{u}_{a}, \Upsilon_{C}^{\star}\right)\right) \\
-0.5 \delta t \Theta^{\star} \widetilde{\mathbf{G}} \nabla \varphi \equiv \widehat{\widehat{\mathbf{u}}}-0.5 \delta t \Theta^{\star} \widetilde{\mathbf{G}} \nabla \varphi
\end{aligned}
$$

which symbolises a system of three linear algebraic equations with three unknown components of the velocity vector $\mathbf{u}$ at each point of the colocated grid. Viewing the lhs of (10) as a linear operator $\mathbf{L}$ acting on the velocity vector $\mathbf{u}$,

$$
\mathbf{L} \mathbf{u}=\widehat{\widehat{\mathbf{u}}}-0.5 \delta t \Theta^{\star} \widetilde{\mathbf{G}} \nabla \varphi
$$

the closed-form expression for the velocity update may be symbolised as

$$
\mathbf{u}=\check{\mathbf{u}}-\mathbf{C} \nabla \varphi
$$

where $\check{\mathbf{u}}=\mathbf{L}^{-1} \widehat{\widehat{\mathbf{u}}}$ and $\mathbf{C}=\mathbf{L}^{-1} 0.5 \delta t \Theta^{\star} \widetilde{\mathbf{G}}$ denotes a $3 \times 3$ matrix of known coefficients. The expanded forms of (12) in either tensorial or explicit component notation can be found in [109] and [135,137], respectively. Noting that the potential temperature perturbation $\theta^{\prime}$ is updated according to (6) upon the final velocity update, the only lacking element to complete the solution at each iteration is the pressure perturbation, and this leads to elliptic boundary value problems (BVPs) for $\varphi$.

\subsection{Elliptic boundary value problems}

For the soundproof PDEs, formulating the BVPs is straightforward, because the underlying theory of the soundproof systems implies specific velocity divergence constraints $[84,35]$. Recalling from section 2 that in stationary coordinates $\mathbf{v}=\widetilde{\mathbf{G}}^{T} \mathbf{u}$, (12) entails

$$
\mathbf{v}=\check{\mathbf{v}}-\widetilde{\mathbf{G}}^{T} \mathbf{C} \nabla \varphi
$$

so the velocity divergence constraints amount to Poisson BVPs for $\varphi$,

$$
\begin{aligned}
& \nabla \cdot \varrho^{*} \theta\left(\check{\mathbf{\mathbf { v }}}-\widetilde{\mathbf{G}}^{T} \mathbf{C} \nabla \varphi\right)=\varrho^{*} \mathcal{H}, \\
& \nabla \cdot \varrho^{*}\left(\check{\check{\mathbf{v}}}-\widetilde{\mathbf{G}}^{T} \mathbf{C} \nabla \varphi\right)=0,
\end{aligned}
$$

respectively, for the pseudo-incompressible and anelastic PDEs. ${ }^{10}$

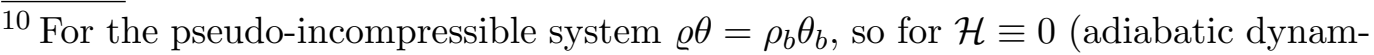
ics), the pseudo-incompressible density can be replaced altogether with $\rho_{b} \theta_{b} / \theta_{0}$ and (14a) with (14b), in the spirit of the anelastic system [135,138].
} 
For the compressible PDEs, the BVPs are optional in that they are dictated by the extended stability of numerical schemes. In our NFTFV framework, the evolutionary form of the equation of state, derived by taking $d / d t$ of (2), couples all thermodynamic variables and the flow field (through $d \varrho / d t$ and the mass continuity equation), thus providing a constraint for pressure and velocity in (12). Taking $d / d t(2)$, expressing the result in the conservation law form, and manipulating the terms $[138,68]$ leads to the PDE

$$
\frac{\partial \varrho^{*} \varphi}{\partial t}+\nabla \cdot\left(\varrho^{*} \mathbf{v} \varphi\right)=\varrho^{*} \sum_{\ell=1}^{3}\left(\frac{a_{\ell}}{\zeta_{\ell}} \nabla \cdot \zeta_{\ell}\left(\check{\mathbf{v}}-\widetilde{\mathbf{G}}^{T} \mathbf{C} \nabla \varphi\right)\right)+b \varphi+c,
$$

where coefficients $a_{\ell}, b, c$ may depend on $\varphi$ but the modified densities $\zeta_{\ell}$ are explicitly known. Interpreting (15) as an archetype PDE (3), and integrating it to $\mathcal{O}\left(\delta t^{2}\right)$ with a mixed forward/backward template

$$
\varphi_{\mathbf{i}}^{n+1}=\mathcal{A}_{\mathbf{i}}\left(\widetilde{\varphi}, \mathbf{V}^{n+1 / 2}, \varrho^{* n}, \varrho^{* n+1}\right)+\left.\delta t \widetilde{R}^{\varphi}\right|_{\mathbf{i}} ^{n+1} \equiv \widehat{\varphi}+\left.\delta t \widetilde{R}^{\varphi}\right|_{\mathbf{i}} ^{n+1},
$$

where $\widetilde{\varphi}=(\varphi+\delta t(b \varphi+c))^{n}$ and $\widetilde{R}^{\varphi} \equiv \operatorname{rhs}(15)-(b \varphi+c)$, provides a discrete implicit constraint for (12)

$$
0=-\sum_{\ell=1}^{3}\left(\frac{A_{\ell}^{\star}}{\zeta_{\ell}} \nabla \cdot \zeta_{\ell}\left(\check{\mathbf{v}}-\widetilde{\mathbf{G}}^{T} \mathbf{C} \nabla \varphi\right)\right)-B^{\star}(\varphi-\widehat{\varphi}) .
$$

The coefficients $A^{\star}$ and $B^{\star}$ in (17) effect from coefficients $a_{\ell}$ in (15) and the superscript ${ }^{*}$ indicates that their dependence on $\varphi$ is lagged. The Helmholtz problem (17) was discussed in $[138,68]$. In the NFTFV codes, we solve both (14) and (17) with a bespoke nonsymmetric preconditioned Generalised Conjugate Residual (GCR) approach, widely discussed in the literature; cf. [135] for a recent overview and a comprehensive list of references.

\subsection{Spatial discretisation}

The mathematical formalism adopted in sections 2 and 3 together with the colocated arrangement of dependent variables simplifies the presentation of the discrete spatial differential operators. The finite-volume discretisation follows the median-dual approach described in [150]. It is briefly summarized below.

For clarity, Fig. 1 shows a schematic of an arbitrary mesh in 2D. The mediandual FV approach constructs the control volume containing the node $i$ by joining the barycentres of polygonal mesh cells encompassing the node $i$ with the midpoints of the edges originating in the node $i$. The derivations and details of median-dual unstructured mesh discretisation of differential operators entering NFTFV schemes and the MPDATA operator are provided in [131] and [148], and a recent summary of finite-volume MPDATA is provided in 


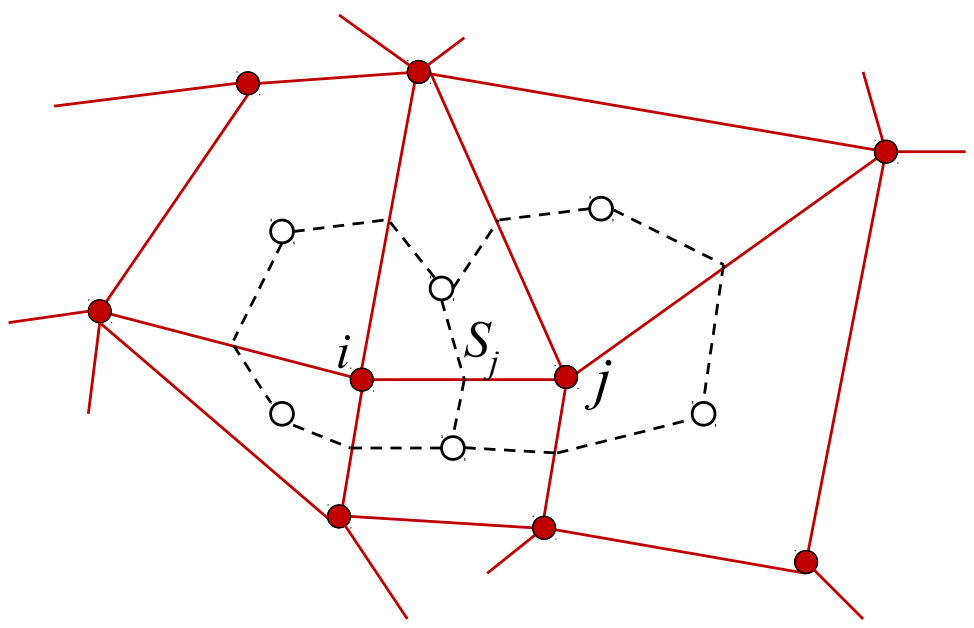

Fig. 1. The edge-based, median-dual approach in 2D. The edge connecting nodes $i$ and $j$ of the primary polygonal mesh pierces, precisely in the edge centre, the face $S_{j}$ shared by computational dual cells surrounding nodes $i$ and $j$; open circles represent barycentres of the primary mesh, while solid and dashed lines mark primary and dual meshes, respectively.

[152]. Here, we highlight a key tool for designing discrete differential operators for control-volume schemes. For a differentiable vector field $\mathbf{A}$, the Gauss divergence theorem- $\int_{\Omega} \nabla \cdot \mathbf{A}=\int_{\partial \Omega} \mathbf{A} \cdot \mathbf{n}$-applied over the control volume $\mathcal{V}_{i}$ surrounding node $i$ leads to

$$
\nabla_{i} \cdot \mathbf{A}=\frac{1}{\mathcal{V}_{i}} \sum_{j=1}^{l(i)} A_{j}^{\perp} S_{j}
$$

Hereafter, $l(i)$ numbers edges connecting node $i$ with its neighbours $j$, and $S_{j}$ refers both to the face per se and its surface area. Equation (18) is exact given $\nabla_{i} \cdot \mathbf{A}$ is interpreted as the mean value of $\nabla \cdot \mathbf{A}$ within the volume $\mathcal{V}_{i}$, while $A_{j}^{\perp}$ is interpreted as the mean normal component of the vector $\mathbf{A}$ at the cell face $S_{j}$. The approximation begins with specifying $A_{j}^{\perp}$ in terms of data available on the mesh; i.e., in terms of mean values of the field within the control volumes $\mathcal{V}_{i}$ and $\mathcal{V}_{j}$. One elementary example is

$$
A_{j}^{\perp}=0.5 \mathbf{n}_{j} \cdot\left[\mathbf{A}_{i}+\mathbf{A}_{j}\right],
$$

where $\mathbf{n}_{j}$ is a mean outward unit normal to the face $S_{j}$. Partial derivatives $\partial \Phi / \partial x^{I}$ of a scalar field $\Psi$ can also be interpreted in terms of the Gauss theorem (by representing the derivative as the divergence of the augmented vector field $\left.\Phi \nabla x^{I}\right)$ as, e.g.,

$$
\left(\frac{\partial \Phi}{\partial x^{I}}\right)_{i}=\frac{1}{\mathcal{V}_{i}} \sum_{j=1}^{l(i)} 0.5\left(\Phi_{i}+\Phi_{j}\right) S_{j}^{I}
$$

where $S_{j}^{I}$ denotes the $I$ th component of the oriented surface element $\mathbf{S}_{j}=S_{j} \mathbf{n}_{j}$ 
of the face at the $j$ th edge.

\section{RESULTS}

In the following we substantiate the theoretical considerations of the preceding sections with applications addressing distinct classes of canonical atmospheric flows. The first example quantifies the approach fidelity in simulation of a small-scale convective planetary boundary layer over flat terrain. The second example addresses influence of complex terrain on mesoscale atmospheric motions in simulation of a strongly stratified flow past a steep isolated hill. The third example is concerned with non-Boussinesq amplification and breaking of deep mesoscale stratospheric gravity waves. Finally, the fourth example addresses global baroclinic instability, an archetype of global atmospheric circulation. Then we also address the computational efficiency of the approach.

\subsection{Convective boundary layer}

Following [137] we show the numerical experiment from [91], which was one of the first systematic demonstrations of the implicit large-eddy-simulation (ILES) with MPDATA based NFTFD schemes. The generalised equations (1) assume a nonrotating Boussinesq limit of the anelastic equations, with constant reference profiles $\theta_{b}(z)=\theta_{0}$ and $\rho_{b}(z)=\rho_{0}$ on a horizontally periodic small-scale Cartesian domain. The growth of the convective boundary layer is driven by a prescribed diabatic source $\mathcal{H}$ in (1b) that assumes divergence of an exponentially decaying surface heat flux over a few mesh increments in the vertical. The effect of surface friction in (1c) is parametrized similarly, as $\boldsymbol{D}=$ $-d \boldsymbol{\tau} / d z$, with exponentially decaying stress $\boldsymbol{\tau}$ with surface value defined by a simple drag law; see [137] for details. Because these specified diabatic/viscous forcings quickly decay with height, they only parametrize near-surface effects; whereas subgrid-scale modelling aloft is delegated to dissipative properties of MPDATA $[110,107]$.

The model domain, $L x \times L y \times L z=3200 \times 3200 \times 1500 \mathrm{~m}^{3}$, is periodic in the horizontal, and a stress-free rigid-lid boundary is assumed at the top. Gravity wave absorbers attenuate the solution toward ambient conditions in the vicinity of the upper boundary. As an initial condition, a $500 \mathrm{~m}$ deep well-mixed ambient layer is prescribed, with a constant temperature $\theta_{a}(z)=$ $\theta_{0}=300 \mathrm{~K}$. Aloft, $\theta_{a}(z)=\theta_{0}\left[1+z N^{2} / g\right]$ and the Brunt-Väisällä frequency $N=10^{-2} \mathrm{~s}^{-1}$ define stable stratification $S=N^{2} / g$. The ambient wind field $\mathbf{u}_{a}=0$. The initial conditions are generated by perturbing $\theta_{a}$ and vertical velocity component, $w$, with small amplitude white noise and then finding the 
potential flow consistent with incompressible mass continuity in (1a) for the Boussinesq limit. Simulations were run for 15000 s. A statistically stationary state is reached after about $9000 \mathrm{~s}$, which is equivalent to about 8 large-eddy turnover time scales. Three basic simulations were performed for this study.
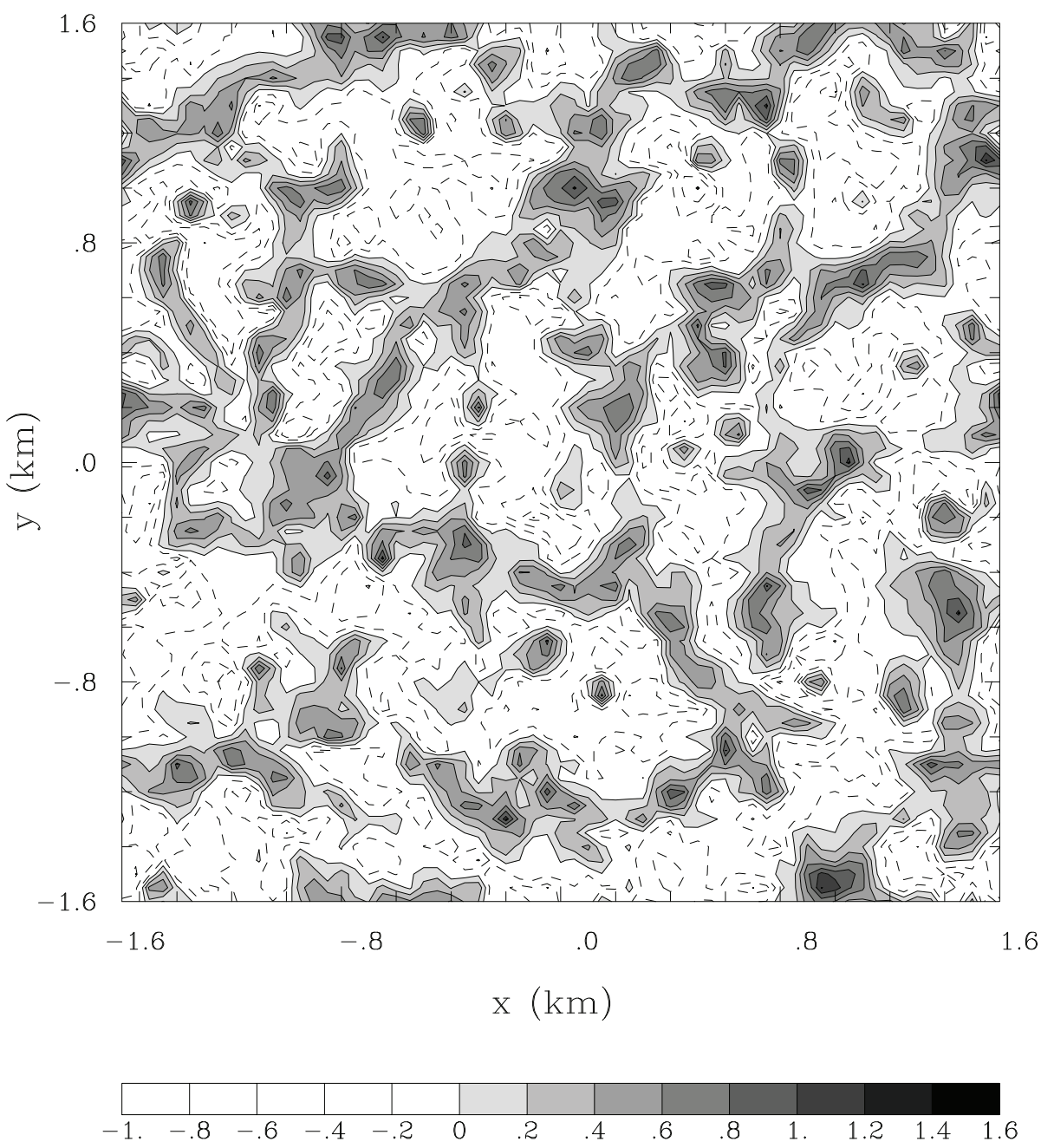

Fig. 2. Vertical velocity in the horizontal plane $150 \mathrm{~m}$ above the heated surface; an instantaneous solution for triangular prismatic mesh is shown after $\sim 13$ eddy turnover times; dashed contours indicate negative values.

The reference simulation, Run $\mathbf{R}$ uses the structured-grid NFTFD code [110] with $N x \times N y \times N z=64 \times 64 \times 51$ regularly distributed points of a Cartesian grid. Two simulations conducted with the NFTFV code, Run G and Run $\mathbf{T}$ use, respectively, the Cartesian grid of Run $\mathbf{R}$ and the unstructured mesh. The primary unstructured mesh consists of $N x y \times N z=5228 \times 50$ triangular based prisms, stacked in 50, $\delta z=30 \mathrm{~m}$ thick, layers. Figure 2 displays the instantaneous vertical velocity field at the end of the Run $\mathbf{T}$, organized into characteristic, albeit irregular, Rayleigh-Bénard cells [107]. The quantitative analysis in [137] documents good agreement of all experiments. 

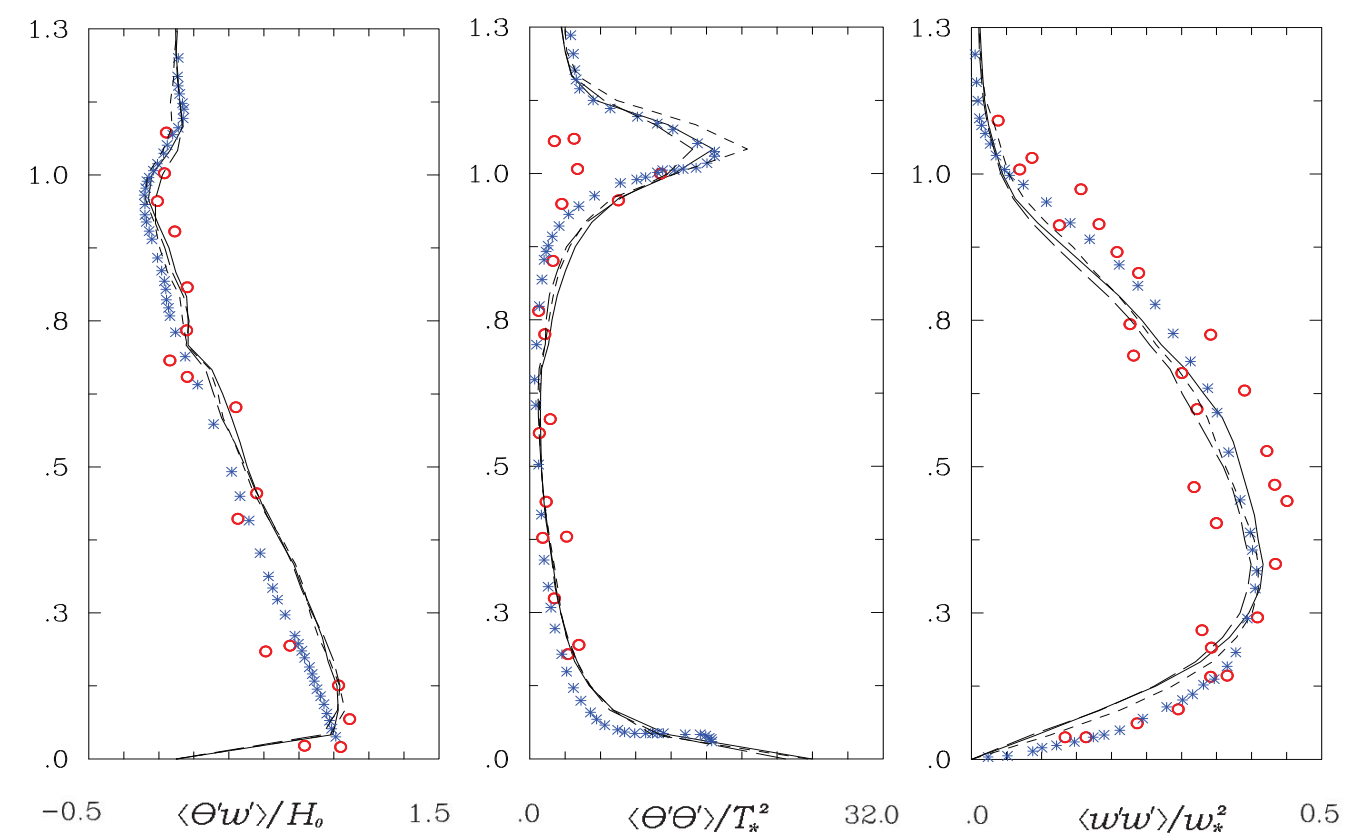

Fig. 3. Vertical profile of dimensionless resolved heat flux, and variances of temperature as well as vertical velocity in Runs $\mathbf{T}$ (solid), G (long dashes), and $\mathbf{R}$ (short dashes), with dimensionless height on the ordinates; blue crosses denote LES result of [115], and red circles represent field and laboratory data.

To illustrate the quality of the NFTFV simulations, the normalized vertical profiles of resolved heat flux $\left\langle\theta^{\prime} w^{\prime}\right\rangle$, temperature variance $\left\langle\theta^{\prime} \theta^{\prime}\right\rangle$, and vertical velocity variance $\left\langle w^{\prime} w^{\prime}\right\rangle$ (here primes denote deviation from the horizontal average $\langle\cdot \cdot\rangle)$ are compared in Fig. 3 with the benchmark LES simulations of, and superimposed measurements by, Schmidt and Schumann [115]. Generally, the profiles generated with the NFTFV codes match closely each other and agree within the data scatter with the structured-grid NFTFD calculations and LES results of [115].

\subsection{Strongly stratified flow past a steep isolated hill; simulation on tetrahedral meshes}

In contrast to modelling engineering flows, where simulations on tetrahedral and related meshes have achieved a high degree of maturity, their applicability to atmospheric flows is still being explored. While prismatic meshes are ideally suited for global models - for which the (relatively) thin atmosphere imposes stringent constraints on the design of numerical models - the unstructured tetrahedral discretisation can benefit small- and mesoscale models, where applications involving terrain so complex as caves and canyons cannot be easily resolved with continuous mappings $[40,20,171]$. On the other hand, atmospheric flow simulations pose new challenges for tetrahedral discretisation, because the underlying hydrostatic balance and physics (e.g., rainfall and ra- 
diation) are predominantly ordered in the vertical direction. Following [152] we show the results attesting to the accuracy of tetrahedral discretisation, in the context of strongly stratified flow past a steep 3D mountain. For this purpose, we adopt the canonical problem of a low-Froude number flow past an axially-symmetric hill $[56,125,37]$, simulated in [137] on dual meshes derived from structured and prismatic primary meshes.
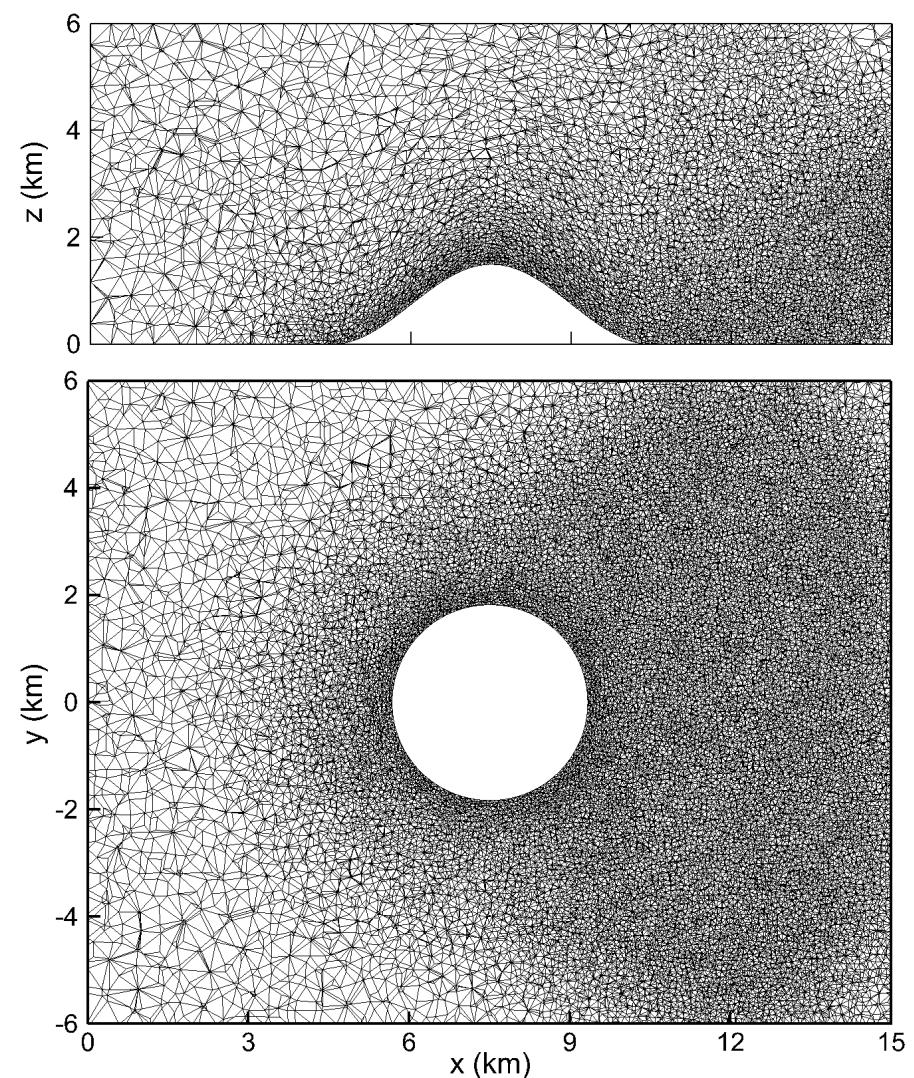

Fig. 4. The central, $y=0$, vertical cross-section (top) of the tetrahedral mesh; the nearby mesh points are projected on the cross-section for visualisation. The horizontal cross-section (bottom) of the mesh at the elevation $z=(1 / 3) h_{0}$.

The cosine hill defined as

$$
\begin{aligned}
& h(x, y)=h_{0} \cos ^{2}(\pi r / 2 L) \text { if } r=\left(\left(x-x_{0}\right)^{2}+\left(y-y_{0}\right)^{2}\right)^{1 / 2} \leq L \\
& h(x, y)=0 \text { otherwise }
\end{aligned}
$$

where the half-width $L=3000 \mathrm{~m}$ and height $h_{0}=1500 \mathrm{~m}$, is centred at the bottom of the computational domain. The domain size is $5 L \times 4 L \times 2 L$ in $x, y$ and $z$ directions, respectively. Two primary meshes consisting of tetrahedral elements were generated in the domain. The first one (not shown) using a uniform background point spacing of $\delta x=\delta y=\delta z=120 \mathrm{~m}$ giving 1945090 of the total number of points, and the second one with the varying point spacing. Figure 4 shows two cross-sections of the primary mesh that consists of 337510 
points and uses varying resolution ranging from $450 \mathrm{~m}$ at the boundaries to about $100 \mathrm{~m}$ in the hill's vicinity. The number of points in the varying resolution tetrahedral mesh is not only substantially lower than in the constant resolution tetrahedral mesh but also lower than in the prismatic (refined in the horizontal only) and Cartesian meshes used for this problem in [137]. In order to produce solutions of matching quality, those meshes consisted of 692533 and 1121812 computational points respectively.

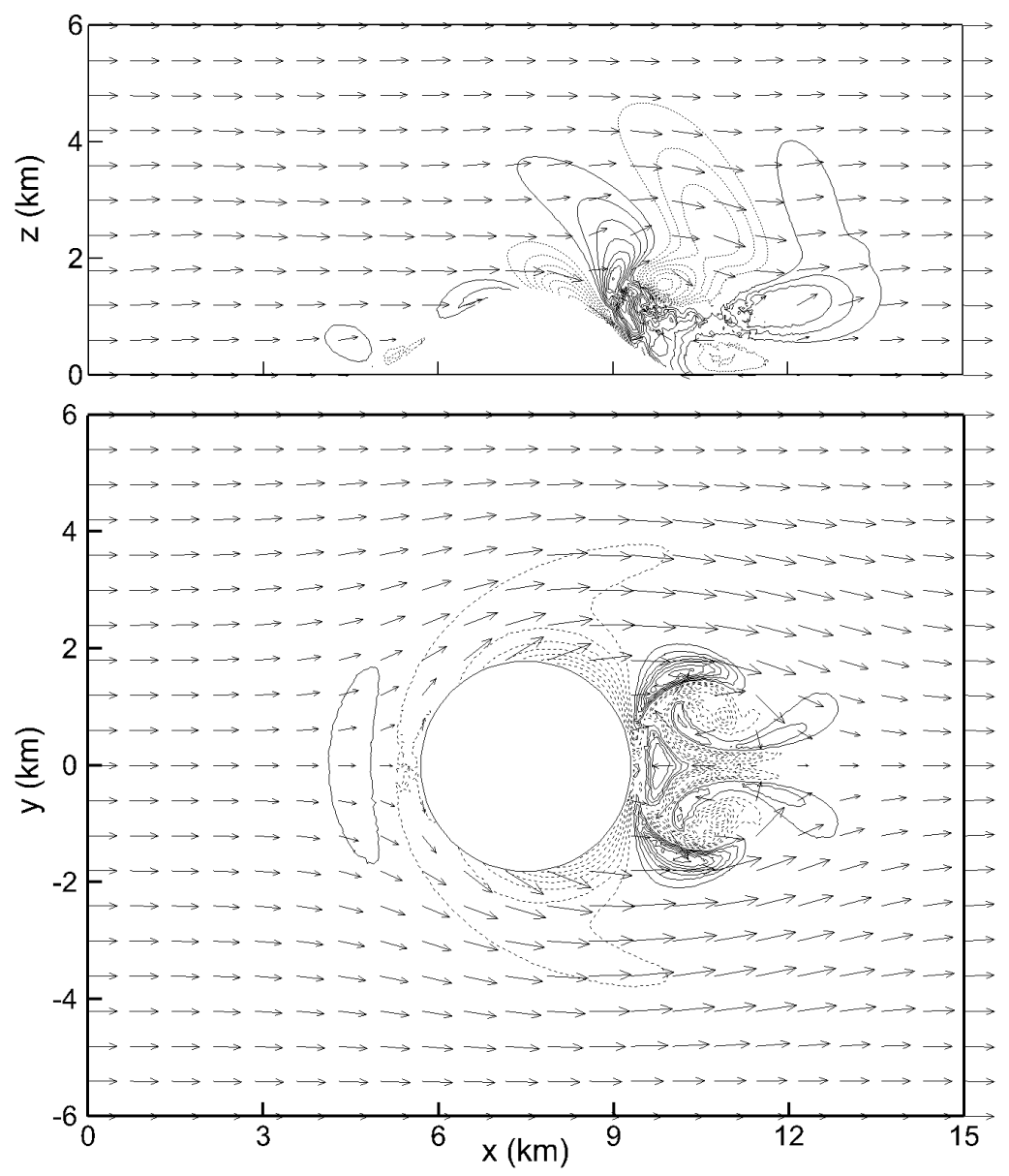

Fig. 5. $F r=1 / 3$ flow solution after two advective time scales $T=L / U$. Contours of vertical velocity in central $x z$ cross section $y=0$ (top) and in the $x y$ cross-section at $z=h_{0} / 3$ (bottom). The contour interval is $0.5 \mathrm{~ms}^{-1}$, and positive/negative contours are presented with solid/dashed lines; the zero contours are not displayed.

The governing equations (1) assume Boussinesq limit of the anelastic PDEs, with the constant potential temperature of the reference state $\Theta_{o}=300 \mathrm{~K}$ and the ambient state characterised by constant buoyancy frequency $N=$ $10^{-2} s^{-1}$ and uniform wind $U=5 \mathrm{~ms}^{-1}$. With the specified hill geometry, the ambient conditions result in a low Froude number, $F r=U / N h_{0}=1 / 3$, flow. Because $h_{0} / L \sim \mathcal{O}(1)$, the problem is essentially nonhydrostatic and can be compared to experimental results given in [56]. All results are shown after two advective time scales $T=L / U(t=1200 \mathrm{~s})$ when the main features 
of the solution are already established. The initial condition is provided by the solution of the potential flow problem, with a gradient of the potential perturbation imposed on the ambient wind. While the boundary conditions are rigid in $x, y$ and $z$, the gravity-wave absorbers near the upper and lateral streamwise boundaries attenuate the solution toward ambient profiles with absorbing coefficient increasing linearly from zero at the distance $L / 2$ from the boundary to $150^{-1} \mathrm{~s}^{-1}$ at the boundary.

The flow patterns displayed in figure 5 are computed for the varying resolution mesh. They show key features of a low Froude number flow, including the characteristic separation and reversal of the lower upwind stream, and the formation of intense vertically-oriented vortices on the lee side of the hill [56,37], with the flow aloft transitioning to the linear gravity wave response [121]. In Figure 5, in the central $x z$ cross-section at $y=0$, a turbulent wake is formed in the lee-side of the hill and characteristic gravity waves response is visible above the wake. This result matches closely the reference solution obtained on structured and prismatic meshes (upper panels in Figs. 6 and 8 in [137]). Figure 5, in $x y$ cross-section at $z=(1 / 3) h_{0}=500 \mathrm{~m}$, presents a pair of eddies behind the hill, showing the intrinsic three-dimensionality of the lee-side flow, the horizontal flow pattern of which also compares well with the reference results in [137]. In brief, the reason of this flow structure is that the incoming flow up to $z_{c} \approx(1-F r) h_{0}$ - the so called dividing streamline [56] - is forced to deflect and split around the hill as it lacks sufficient kinetic energy to go over the hill. Above the dividing streamline the hill is sufficiently low for the flow to go over, thus resulting in the characteristic gravity response aloft. The corresponding solutions for the regular tetrahedral mesh are not shown, because their departures from the results in Fig. 5 are insignificant. For quantitative analysis attesting to the quality of the NFTFV results the interested reader is referred to [152] and references therein.

\subsection{Amplification and breaking of $2 D$ stratospheric gravity wave}

The preceding examples addressed small- and mesoscale problems in a relatively shallow portion of the troposphere, where incompressible Boussinesq limit of the anelastic equations holds well; i.e., the differences between the results generated with either of the PDEs included in (1) are insignificant [27]. Following [135], here we consider an essentially non-Boussinesq problem in a 2D deep stratosphere. A small amplitude wave packet - excited by a hypothetical squall line with the top impinging upon the tropopause-propagates into the stratosphere. Because density of the media decreases with altitude, the amplitude of the wave increases with height in proportion to $\rho_{b}^{-1 / 2}$. When the wave amplitude becomes comparable with the vertical wavelength, the problem becomes inherently nonlinear. Then, the wave overturns and breaks 


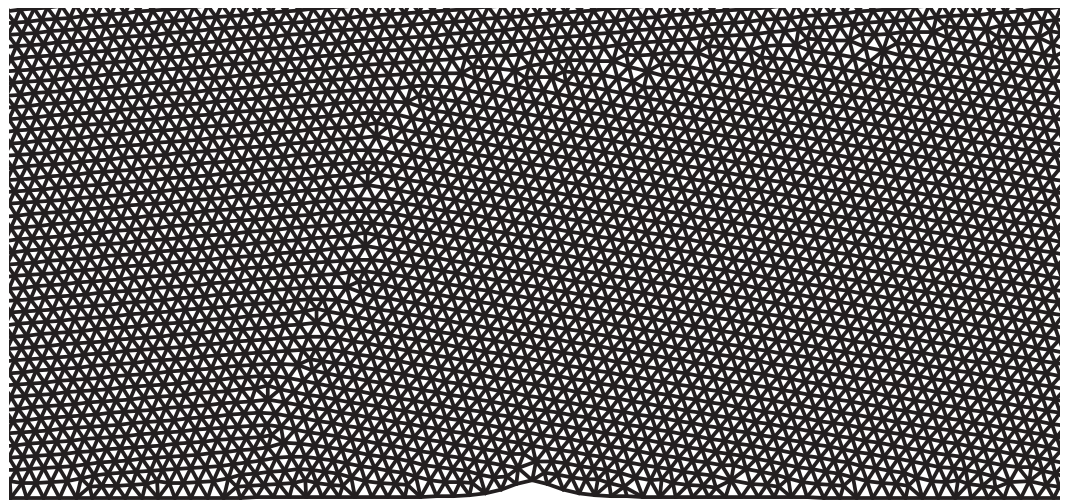

Fig. 6. A near surface fragment of the primary mesh used in simulations of a deep stratospheric gravity; the height and half-width of the lower boundary deflection are 628 and $1000 \mathrm{~m}$, respectively.

generating bursts of turbulence far from the excitation region. The problem is numerically challenging, because it covers about nine density height scales and vertical wavelengths, and a transition from the linear-wave regime near the bottom of the domain to a vigorous turbulent flow with a broad range of scales about $30 \mathrm{~km}$ aloft.
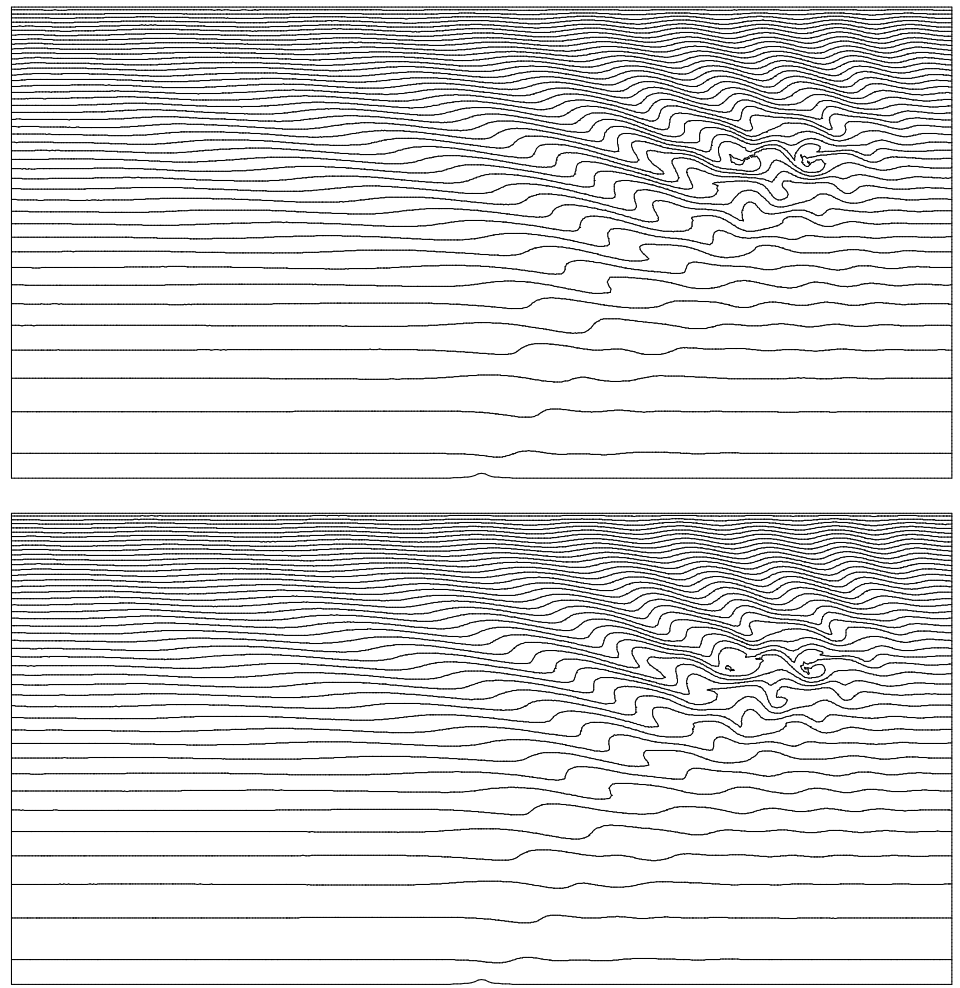

Fig. 7. Isentropes at $t=90 \mathrm{~min}$, in the entire domain, simulated with the pseudo-incompressible (top) and anelastic (bottom) PDEs using the unstructured mesh highlighted in Fig. 6.

Following [135] the model setup assumes an isothermal stratosphere, with uniform potential temperature stratification fourfold of the tropospheric val- 
ues. The background density decreases exponentially, such that the density scale $H_{\rho}=-\left(d \ln \rho_{b} / d z\right)^{-1}=6515 \mathrm{~m}$ is 3.5 times smaller than the potential temperature scale $H_{\theta}$. The ambient wind $\mathbf{u}_{a}=\left(u_{a}, 0\right)$ is constant with speed $u_{a}=U=20 \mathrm{~ms}^{-1}$, and the ambient profile of potential temperature $\theta_{a}(z) \equiv \theta_{b}(z)$. The $60 \mathrm{~km}$ deep and $120 \mathrm{~km}$ wide domain is resolved with fully unstructured triangular primary mesh illustrated in Fig. 6 that consists of $59 \cdot 10^{3}$ nodes, effecting in a fairly uniform mesh resolution of $\approx 380 \mathrm{~m}$. The wave is excited by a small deflection of the lower boundary with the height profile $h(x)=h_{o}\left[1+(x / L)^{2}\right]^{-1}$ centred at the origin of the $[-60 L, 60 L] \times[0,60 L](x, z)$-domain; the deflection's height and half-width are, respectively, $h_{o}=628.319 \mathrm{~m}$ and $L=1000 \mathrm{~m}$. The problem is inherently nonhydrostatic because the dominant horizontal wavenumber of the problem, $1 / L$, equals the asymptotic wavenumber $N / U$ of the induced waves; where $N$ denotes the buoyancy frequency. Furthermore, the problem is only weakly nonlinear (the Froude number $F r=U / N h_{o} \approx 1.6$ ) with respect to linear Boussinesq theory. The onset of wave breaking in the upper half of the model domain is observed after $90 \mathrm{~min}$ of the simulated time. The corresponding pseudo-incompressible and anelastic solutions are shown in top and bottom panels of Fig. 7. The results convey the same underlying physics while deviating only in minor details, consistent with the results reported in [138] for structured-grid NFTFD model.

\subsection{Baroclinic instability}
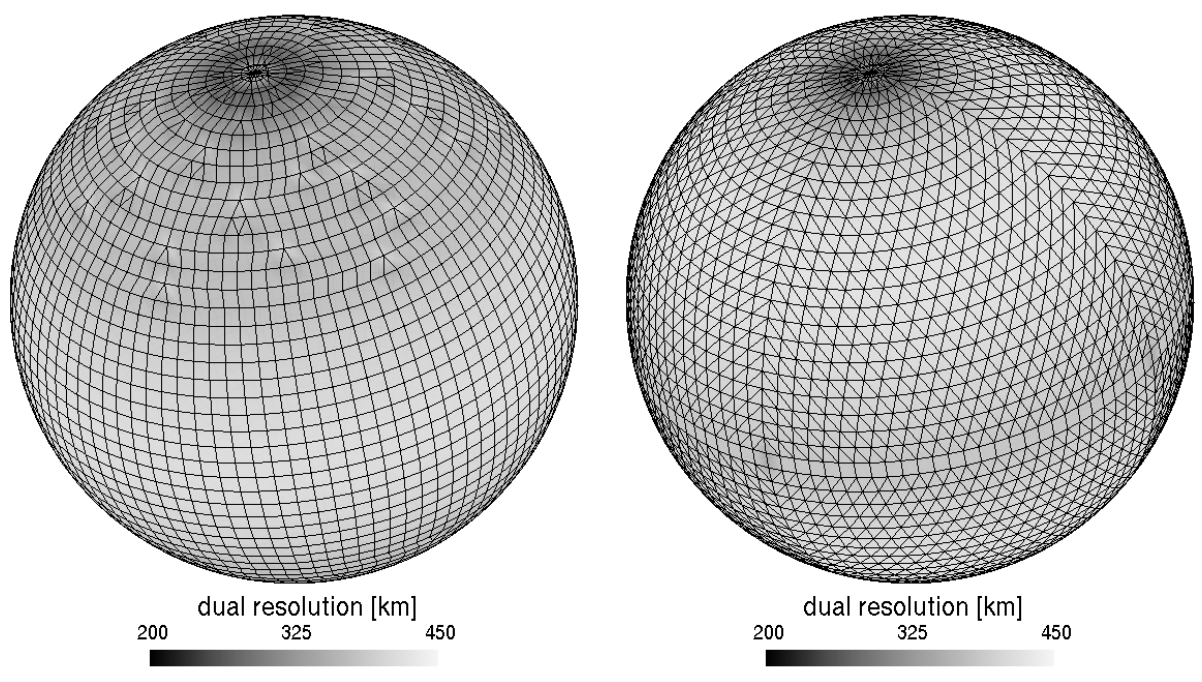

Fig. 8. Classical (left) and octahedral (right) meshes generated around reduced Gaussian grid points with approximate resolution of $3.75^{\circ}(415 \mathrm{~km})$. The shading represents the dual resolution, computed as the square root of the local dual volume.

In contrast to the preceding sections that addressed nonhydrostatic perfor- 
mance of the NFTFV approach in local-area simulations, here we illustrate its capability for simulating essentially hydrostatic modes of global circulation. The illustration employs a high performance finite-volume module (FVM) [139], with NFTFV discretisation hybridising unstructured-mesh in the horizontal with the structured-grid in the vertical. The governing equations are formulated in the classical meteorological latitude-longitude spherical framework $[109,150,138]$ frequently criticised for its notorious stiffness in the polar regions. Yet, unstructured flexible meshes can circumvent this issue by designing dual cells in lat-lon space such as to generate homogeneous meshing on the physical surface of the sphere [150]. In the FVM such bespoke unstructured meshes, Fig. 8, generated about Gaussian grids of the ECMWF Integrated Forecasting System (IFS) - a world leading medium-range spectral-transform based model - circumvent this stiffness and accommodate both spectral-transform and grid-point solutions at the same physical locations. In the vertical, a uniform discretisation in the computational space facilitates the solution of intricate elliptic problems in thin spherical shells, while the pliancy of the physical vertical coordinate is delegated to generalised continuous transformations between computational and physical space. Consequently, the FVM uses the entire analytic apparatus of section 2 .

Here we show the compressible solution generated with FVM using an octahedral reduced Gaussian grid, with horizontal grid spacing of $12.5 \mathrm{~km}$ corresponding to state-of-the-art operational NWP. ${ }^{11}$ The model depth $\approx 24 \mathrm{~km}$ is resolved with 61 stretched vertical levels, with smoothly varying resolution of $\delta z=50 \mathrm{~m}$ near the ground, through $\delta z=150 \mathrm{~m}$ at $2 \mathrm{~km}$ altitude, up to $\delta z=850 \mathrm{~m}$ near the model top. The employed time step $\delta t=80 \mathrm{~s}$ resulted in the maximal Courant number $\gtrsim 0.5$ at the time of the frontal collapse.

Figure 9 displays the baroclinic wave train after 8 days evolution from a weakly perturbed unstable-equilibrium initial state consisting of the two planetary jets in midlatitudes. The upper panel shows isentropes overlaid with contours of the vertical velocity, in the vertical cross section through the centre of the northerly jet. Lower panel shows isentropes together with the surface meridional velocity. Together these figures highlight a 3D structure of idealised frontogenesis and formation of weather systems in midlatitudes. These results are consistent with those familiar from the literature. Notably, at the relatively high resolution employed, the simulation begins to capture mesoscale gravity waves radiated at collapsing fronts. In inviscid simulations the associated grid-scale features are intermittent. Generally they are filtered out with an aid of artificial viscosity or subgrid-scale parametrisations. Here they are controlled by the model nonoscillatory numerics in the spirit of ILES. The latter is substantiated in Fig. 10 depicting surface kinetic energy spectra at

\footnotetext{
$\overline{{ }^{11} \text { Soon }}$ after these calculations were conducted, the state-of-the-art resolution got refined down to $9 \mathrm{~km}[89]$.
} 


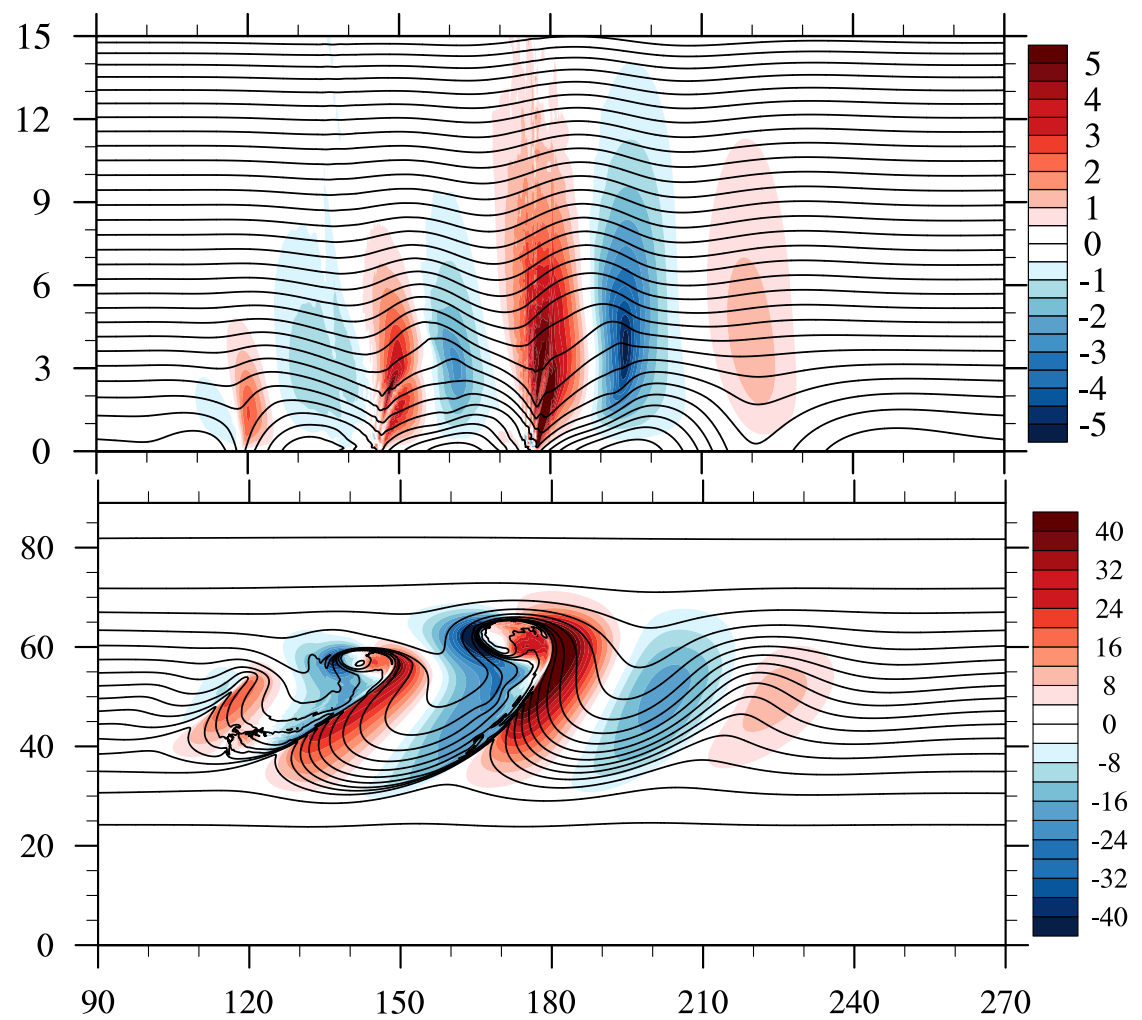

Fig. 9. Baroclinic instability, day 8: (top) color map of vertical velocity $[\mathrm{cm} / \mathrm{s}]$ overlaid with isentropes, in the vertical cross section at the $53^{\circ} \mathrm{N}$ latitude; (bottom) color map of surface meridional velocity $[\mathrm{m} / \mathrm{s}]$ and isentropes. In both panels, isentropes are displayed with the contour interval of $5 \mathrm{~K}$. The abscissa and ordinate of the lower panel are in degrees, whereas the ordinate of the upper panel is in kilometres.

various resolutions; cf. Fig. 8 in [175] for similar results generated with five different nonhydrostatic models. The spectra follow each other closely at the resolved scales of motion and tend to separate at the grid-scale, where implicit dissipation is effective. For an extended discussion of spectra generated in the evolution of baroclinic waves, see [69] and references therein.

We end this section with a comment on the potential efficiency of the NFTFV approach. The calculations in preceding sections were all serial and their overall expense were insignificant. On the other hand, the FVM shares the equal regions parallelisation scheme with the IFS, with multiple layers of parallelism hybridising MPI tasks and OpenMP threads. The presented simulations of the baroclinic instability were conducted on Cray XC30 at the ECMWF and used 768 MPI tasks and 6 OMP threads (i.e., 4608 cores). An equivalent simulation (not shown) with the $30 \mathrm{~km}$ resolution and three times larger time step $\delta t=240$ s required roughly 20 times shorter wall-clock time of the 4608 cores, commensurate with 17 -fold reduction of the computational work due to the coarser temporal and spatial resolution. This together with the quality of the results indicates the potential of NFTFV approach for advanced NWP and 


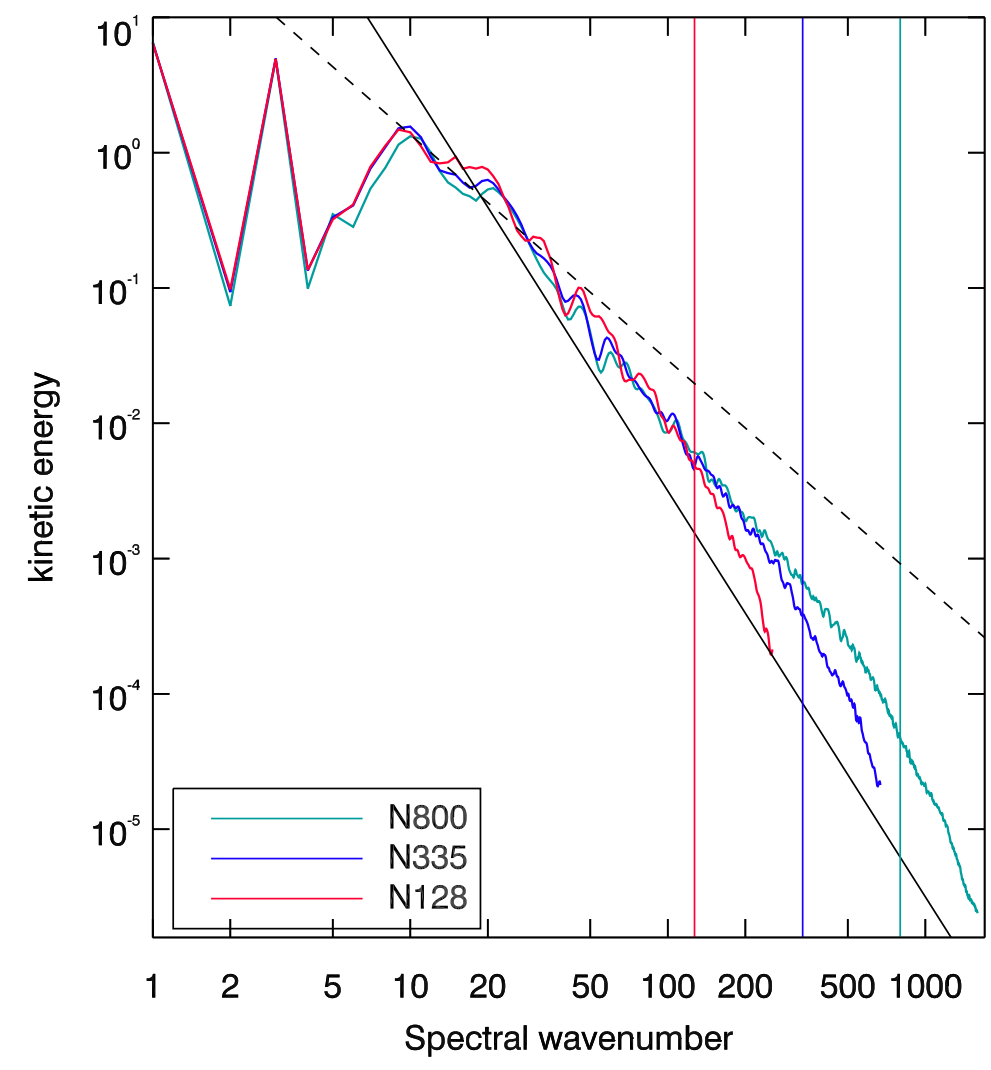

Fig. 10. Surface kinetic energy spectra at day 9 of the baroclinic instability evolution, simulated with various horizontal resolutions. Vertical lines on the right indicate four grid intervals for each corresponding resolution. For reference, the -3 and $-5 / 3$ slopes are shown with solid and dashed lines, respectively.

climate studies.

\section{Conclusion}

We have reviewed the historical progress leading to the implementation of finite-volume methods in atmospheric modelling, with emphasis on a consistent and systematic development that shapes the numerical formulation well-suited for the atmospheric flows of all scales. As illustrated in section 4, the NFTFV approach has been proven a success in capitalising on the intrinsic strengths of the FV methodology, so as to benefit the resulting numerical model in at least three aspects. First, the rigorous conservativeness guarantees reliable and physics-consistent solutions; second, the adaptability for arbitrary unstructured meshes effectively deals with geometrical complexities and rigid connectivity of structured grids; and third, the semi-implicit solution procedure mitigates CFL restrictions while solving for all scales under a single numerical framework. 
The presented NFTFV approach is technically akin to the general-purpose engineering CFD solvers designed for wide-spectrum of applications, and thus one should think it would be appealing in a wide range of atmospheric scenarios as well as other geophysical/astrophysical disciplines and challenging multiscale problems of industrial CFD. The example of section 4.4 shows that the NFTFV technology can complement established spectral-transform based, semi-implicit semi-Lagrangian NWP models - most effective in medium-range weather forecasting - by supplying them with methods, connectivities, and communication patterns previously inaccessible to these models. This enables combining the strengths of spectral-transform NWP and cloud-resolving research models into a new hybrid entity that offers exciting opportunities for advancing computational meteorology and eventually other areas of computational science and engineering. Nowadays strong arguments are emerging for establishing international supercomputing centres dedicated to climate prediction [106]. In order to reduce uncertainties in global warming, climate simulations are envisaged that would run globally at $1 \mathrm{~km}$ horizontal resolution over a time scale of century. This requires exascale calculations with over $10^{18}$ floating-point operations per second [106]. To achieve such a scale of computing, apart from the new generation computer hardware new numerical algorithms will need to evolve, building on strengths and experience of past and present developments.

The development of dynamical cores for atmospheric general circulation models is an active field of research. For this purpose, nearly all existing numerical methodologies usable throughout interdisciplinary CFD have been experimented with. In the quest for unified all-scale atmospheric models (recall the last paragraph of section 1.1), the top-down and bottom-up paths of extending the spectral range of simulated scales were advancing their preferred integration methods for stiff PDEs, with marked examples including semi-Lagrangian semi-implicit time integrators originated in NWP $[187,28,74,10,117,173]$ on the one hand, and Eulerian split-explicit time stepping methods [114,118,6,119], a heritage of small-scale limited-area models [153,64], on the other. The collection of schemes was further enhanced with various forms of spatial discretisation, including finite differences $[187,28,118,6]$, spectral transforms $[187,10,173]$, finite volumes $[114,119]$, and more recently high-order element based methods such as spectral element [154,42,155,29] and discontinuous Galerkin [102,43,7,44], or the multi-moment finite-volume approach $[17,79,80,18]$, or combinations thereof [158]. The technical literature devoted to the advancement of nonhydrostatic atmospheric models is extensive, and the references provided merely illustrate its diversity. So far there is no approach perfect in every respect, and all existing techniques have some trade-off between computational stability, numerical accuracy, computational complexity and performance $[188,172]$. Consequently, in order to meet the challenge of exascale computing, it is important to see multiple options being advanced, especially that the future may belong to hybrids enabling methods, as exemplified in section 4.4. 


\section{Acknowledgements:}

Helpful comments from Christian Kühnlein, Gianmarco Mengaldo, Nils Wedi and two anonymous referees are gratefully acknowledged. This work was supported by the funding received from the European Research Council under the European Union's Seventh Framework Programme (FP7/2012/ERC Grant agreement no. 320375), and from the ESCAPE project; ESCAPE is funded by the European Commission under the Horizon 2020 Programme - grant agreement 671627 .

\section{Appendix. Specifications of the spherical frame}

Governing PDEs (1) assume the spherical curvilinear framework of [109]. Consequently, the vector $\mathbf{u}$ represents the physical velocity with components aligned at every point of the spherical shell with axes of a local Cartesian frame (subsequently marked as ${ }_{c}$ ) tangent to the lower surface $(r=a)$ of the shell; $r$ is the radial component of the vector radius, and $a$ is the radius of the sphere, cf. Fig. 7.7, section 7.2 in [36]. Consequently, $d x_{c}=r \cos \phi d \lambda, d y_{c}=r d \phi$ and $z_{c}=r-a$; where $\lambda$ and $\phi$ denote longitude and latitude angles, respectively. Then, in the formalism of sections 2 and 3 and in the absence of coordinate stretching, $x=a \lambda, y=a \phi$, and $z=z_{c}$; thereby effectively employing longitude-latitude coordinates standard in many global atmospheric models [150]. Furthermore, the coefficient matrix $\widetilde{\mathbf{G}}$ consists of zero off-diagonal entries, whereas $\widetilde{G}_{1}^{1}=[\Gamma \cos (y / a)]^{-1}, \widetilde{G}_{2}^{2}=\Gamma^{-1}$, and $\widetilde{G}_{3}^{3}=1$. Here, $\Gamma=1+z / a$, and indices 1,2 , and 3 correspond to $x, y$, and $z$ components. Consequently, the Jacobian is $\mathcal{G}=\Gamma^{2} \cos (y / a)$.

In the momentum equation, the components of the Coriolis acceleration are

$$
\begin{array}{r}
-\mathbf{f} \times \mathbf{u}=\left[v f_{0} \sin (y / a)-\right. \\
w f_{0} \cos (y / a), \\
-u f_{0} \sin (y / a), \\
\left.u f_{0} \cos (y / a)\right],
\end{array}
$$

where $\mathbf{u}=[u, v, w]$ and $f_{0}=2|\boldsymbol{\Omega}|$. Furthermore, the metric forcings (viz., component-wise Christoffel terms associated with the convective derivative of the physical velocity) are, 


$$
\begin{aligned}
& \mathcal{M}(\mathbf{u})=(\Gamma a)^{-1}[\tan (y / a) u v-u w, \\
& -\tan (y / a) u u-v w, \\
& u u+v v] \text {. }
\end{aligned}
$$

\section{References}

[1] R. Abgrall, P. Smolarkiewicz, F. Xiao, S. Zaleski, Frontiers in Computational Physics: Modeling the Earth System, J. Comput. Phys., 271 (2014) 1.

[2] B.J. Adler, Introduction, J. Comput. Phys., 135 (1997) 100.

[3] J.L. Anderson, S. Preiser, E. Rubin, Conservation form of the hydrodynamics in curvilinear coordinate systems, J. Comput. Phys. 2 (1968) 279-287.

[4] A. Arakawa, Computational design for long-term numerical integration of the equations of fluid motion: Two-dimensional incompressible flow. Part I, J. Comput. Phys. 1 (1966) 119-143.

[5] D.P. Bacon, N.N. Ahmad, Z. Boybeyi, T.J. Dunn, M.S. Hall, P.C.S. Lee, R.A. Sarma, M.D. Turner, K.T. Waight, S.H. Young, J.W. Zack, A Dynamically Adapting Weather and Dispersion model: The Operational Multiscale Environment Model with Grid Adaptivity (OMEGA), Month. Weather Rev. 128 (2000) 2044-2067

[6] M. Baldauf, A. Seifert, J. Förstner, D. Majewski, M. Raschendorfer, Operational convective-scale numerical weather prediction with the COSMO model: Description and sensitivities, Month. Weather Rev. 139 (2011), 3887-3905.

[7] L. Bao, R. Klöfkorn and R.D. Nair, Horizontally Explicit and Vertically Implicit (HEVI) Time Discretization Scheme for a Discontinuous Galerkin NonHydrostatic Model, Month. Weather Rev., 143 (2015), 972-990.

[8] T.J. Barth, D.C. Jespersen, The design and application of upwind schemes on unstructured meshes, AIAA paper 89-0366, Aerospace Sciences Meeting, 27th, Reno, NV, Jan. 9-12, 1989. 13 p

[9] P. Bauer, A. Thorpe, G. Brunet, The quiet revolution of numerical weather prediction, Nature 525 (2015) 47-55.

[10] P. Bénard, J. Vivoda, J. Mašek, P. Smoliková, K. Yessad, Ch. Smith, R. Brožkovaá, J.F. Geleyn, Dynamical kernel of the ALADIN-NH spectral limited-area model: Revised formulation and sensitivity experiments, Q.J.R. Meteorol. Soc. 136 (2010) 155-169.

[11] H. Bijl, P. Wesseling, A unified method for computing incompressible and compressible flows in boundary-fitted coordinates, J. Comput. Phys. 141 (1998) 153-173. 
[12] J.P. Boris, D.L. Book, Flux-corrected transport. I. SHASTA, a fluid transport algorithm that works, J. Comput. Phys. 11 (1973) 38-69.

[13] C.J. Budd, M.J. Cullen, E.J. Walsh, Monge-Ampere based moving mesh methods for numerical weather prediction, with applications to the Eady problem, J. Comput. Phys. 236 (2013) 247-270.

[14] C.J. Budd, R.D. Russell, E. Walsh, The geometry of r-adaptive meshes generated using optimal transport methods, J. Comput. Phys. 282 (2015) 113137.

[15] Bunge H-P, M.A. Richards, and J.R. Baumgardner, A sensitivity study of threedimensional spherical mantle convection at $10^{8}$ Rayleigh number: Effects of depth-dependent viscosity, heating mode, and an endothermic phase change, J. Geophys. Res. 102 (1997) 11,991-12,007

[16] J.G. Charney, R. Fjørtoft, J. von Neumann, Numerical integration of barotropic vorticity equation, Tellus 2 (1950) 237-254.

[17] C. Chen, F. Xiao, Shallow water model on cubed-sphere by multi-moment finite volume method, J. Comput. Phys. 227 (2008) 5019-5044.

[18] C. Chen, X. Li, X. Shen, F. Xiao, Global shallow water models based on multimoment constrained finite volume method and three quasi-uniform spherical grids, J. Comput. Phys. 271 (2014) 191-223.

[19] A. Chorin, Numerical solution of the Navier-Stokes equations, Math. Comput. 22 (1968) 745-762.

[20] T.L. Clark, A small-scale dynamic model using a terrain-following coordinate transformation, J. Comput. Phys. 24 (1977) 186-215.

[21] T.L. Clark, R.D. Farley, Severe downslope windstorm calculations in 2 and 3 spatial dimensions using anelastic interactive grid nesting - a possible mechanism for gustiness, J. Atmos. Sci. 41 (1984) 329-350.

[22] P. Colella, P.R. Woodward, The piecewise parabolic method (PPM) for gasdynamical simulations, J. Comput. Phys. 54 (1984) 174-201.

[23] J. Côté, C. Jablonowski, P. Bauer, N. Wedi, Numerical methods of the atmosphere and ocean, Chap. 6 in: Seamless prediction of the Earth system: from minute to months, (WMO-No.1156), World Meteorological Organization (2015) 101-124.

[24] C.S. Cotter, P.K. Smolarkiewicz, I.N. Szczyrba, A viscoelastic fluid model for brain injuries, Int. J. Num. Meth. Fluids 40 (2002) 303-311.

[25] W.P. Crowley, Numerical advection experiments, Month. Weather Rev. 96 (1968) 1-11.

[26] M. Cullen, Modelling atmospheric flows, Acta Numerica 16 (2007) 67-154. 
[27] T. Davies, A. Staniforth, N. Wood, J. Thuburn, Validity of anelastic and other equation sets as inferred from normal-mode analysis. Q.J.R. Meteorol. Soc. 129 (2003) 2761-2775.

[28] T. Davies, M.J.P. Cullen, A.J. Malcolm, M.H. Mawson, A. Staniforth, A.A White, N. Wood, A new dynamical core for the Met Office's global and regional modelling of the atmosphere. Q.J.R. Meteorol. Soc. 131 (2005) 1759-1782.

[29] J.M. Dennis, J. Edwards, K.J. Evans, O. Guba, P.H. Lauritzen, A.A. Mirin, A. St-Cyr, M.A. Taylor, P.H. Worley, CAM-SE: a scalable spectral element dynamical core for the community atmosphere model, Int. J. High Perform. Comput. Appl. 26 (2012) 74-89.

[30] J.A. Domaradzki, Z. Xiao, P.K. Smolarkiewicz, Effective eddy viscosities in implicit large eddy simulations of turbulent flows, Phys. Fluids 15 (2003) 38903893.

[31] A. Dörnbrack, J.D. Doyle, T.P. Lane, R.D. Sharman, P.K. Smolarkiewicz, On physical realizability and uncertainty of numerical solutions, Atmos. Sci. Lett. (2005) 118-122.

[32] P.D. Düben, P. Korn, Atmosphere and ocean modeling on grids of variable resolution - a 2D case study, Month. Weather Rev. 142 (2014) 1997-2017.

[33] J.K. Dukowicz, J.D. Ramshaw, Tensor viscosity method for convection in numerical fluid dynamics, J. Comput. Phys. 32 (1979) 71-79.

[34] J.K. Dukowicz, Computational efficiency of the hybrid penaltypseudoincompressibility method for incompressible flow, Comput. Fluids 23 (1994) 479-486.

[35] D.R. Durran, Improving the anelastic approximation. J. Atmos. Sci. 46 (1989) 1453-1461.

[36] J.A. Dutton, The Ceaseless Wind, Dover Publications (1986) pp. 617.

[37] C.C. Epifanio, R. Rotunno, The dynamics of orographic wake formation in flows with upstream blocking, J. Atmos. Sci. 62 (2005) 3127-3150.

[38] G. Fosser, S. Khodayar, P. Berg, Benefit of convection permitting climate model simulations in the representation of convective precipitation, Clim. Dyn. 44 (2015) 45-60.

[39] J.E. Fromm, A method for reducing dispersion in convective difference schemes, J. Comput. Phys. 3 (1968) 176-189.

[40] T. Gal-Chen, C.J. Somerville, On the use of a coordinate transformation for the solution of the Navier-Stokes equations, J. Comput. Phys. 17 (1975) 209-228.

[41] M. Ghizaru, P. Charbonneau, P.K. Smolarkiewicz, Magnetic Cycles in Global Large-eddy Simulations of Solar Convection, Astrophys. J. Lett. 715 (2010) L133-L137. 
[42] F.X. Giraldo, T.E. Rosmond, A scalable spectral element Eulerian atmospheric model (SEE-AM) for NWP: dynamical core tests, Month. Weather Rev., 132 (2004:) 133-153.

[43] F.X. Giraldo, M. Restell, A study of spectral element and discontinuous Galerkin methods for the Navier-Stokes equations in nonhydrostatic mesoscale atmospheric modeling: equation sets and test cases, J. Comput. Phys. 227 (2008) 3849-3877.

[44] F.X. Giraldo, J.F. Kelly, E.M. Constantinescu, Implicit-explicit formulations of a three-dimensional nonhydrostatic unified model of the atmosphere (NUMA), SIAM J. Sci. Comput. 35 (2013) B1162-B1194.

[45] S.K. Godunov, A difference scheme for numerical computation of discontinuous solutions of equations in fluid dynamics, Matematicheskij Sbornink 47 (1959) $540-555$.

[46] S.K. Godunov, Reminiscences about difference schemes, J. Comput. Phys. 153 (1999) 6-25.

[47] W.W. Grabowski, P.K. Smolarkiewicz, A multiscale anelastic model for meteorological research, Month. Weather Rev. 130 (2002) 939-956.

[48] F.F. Grinstein, L. Margolin, W. Rider, Implicit Large Eddy Simulation: Computing Turbulent Fluid Dynamics, Cambridge University Press (2007) pp 546.

[49] H. Guillard, C. Viozat, On the behavior of upwind schemes in the low Mach number limit, Comput. \& Fluids 28 (1999) 63-86.

[50] F. Harlow, E. Welch, Numerical calculation of time-dependent viscous incompressible flow of fluid with a free surface, Phys. Fluids 8 (1965) 21822189 .

[51] F. Harlow, A. Amsden, Numerical calculation of almost incompressible flows, J. Comput. Phys. 3 (1968) 80-93.

[52] F. Harlow, A. Amsden, A numerical fluid dynamics calculation method for all flow speeds, J. Comput. Phys. 8 (1971) 197-213.

[53] A. Harten, High resolution schemes for hyperbolic conservation laws, J. Comput. Phys. 49 (1983) 357-393.

[54] J.D. Hyman, P.K. Smolarkiewicz, C.L. Winter, Heterogeneities of flow in stochastically generated porous media, Phys. Rev. E 86 (2012) 056701.

[55] D. Ham, M. Piggott, T. Ringler, H. Weller, N. Wood, Multiscale Numerics for the Atmospher and Ocean, Isaac Newton Institute for Mathematical Scinces, 22nd August-21st December 2012, https://www.newton.ac.uk/event/amm

[56] C.R. Hunt, W.H. Snyder, Experiments on stably and neutrally stratified flow over a model three-dimensional hill, J. Fluid Mech. 96 (1980) 671-704. 
[57] R. Issa, D. Gosman, A. Watkins, The computation of compressible and incompressible flows by a non-iterative implicit scheme, J. Comput. Phys. 62 (1986) 66-82.

[58] A. Jameson, D. Mavriplis, Finite volume solution of the two-dimensional Euler equations on a regular triangular mesh, AIAA J. 24 (1986) 611-618.

[59] A. Jaruga, S. Arabas, D. Jarecka, H. Pawlowska, P.K. Smolarkiewicz, M. Waruszewski, libmpdata++ 1.0: a library of parallel MPDATA solvers for systems of generalised transport equations, Geosci. Model. Dev. 8 (2015) 10051032 .

[60] C. Karki, S. Patankar, Pressure based calculation procedure for viscous flows at all speed in arbitrary configurations, AIAA J. 27 (1989) 1167-1174.

[61] R. Klein, Semi-implicit extension of a Godunov-type scheme based on low Mach number asymptotics I: One-dimensional flow, J. Comput. Phys. 121 (1995) 213237.

[62] R. Klein, Scale-dependent models for atmospheric flows, Ann. Rev. Fluid Mech. 42 (2010) 249-274.

[63] R. Klein, U. Achatz, D. Bresch, O.M. Knio, P.K. Smolarkiewicz, Regime of validity of soundproof atmospheric flow models, J. Atmos. Sci. 67 (2010) 32263237.

[64] J.B. Klemp, R.B. Wilhelmson, The simulation of three-dimensional convective storm dynamics, J. Atmos. Sci. 35 (1978) 1070-1096.

[65] D.A. Knoll, L. Chacon, L.G. Margolin, V.A. Mousseau, On balanced approximations for time integration of multiple time scale systems, J. Comput. Phys. 185 (2003) 583-611.

[66] C. Kühnlein, P.K. Smolarkiewicz, A. Dörnbrack, Modelling atmospheric flows with adaptive moving meshes, J. Comput. Phys. 231 (2012) 2741-2763.

[67] S. Kumar, R. Bhattacharyya, P.K. Smolarkiewicz, Formation of magnetic discontinuities through viscous relaxation, Phys. Plasmas 21 (2014) 052904.

[68] M. Kurowski, W.W. Grabowski, and P.K. Smolarkiewicz, Anelastic and compressible simulations of moist deep convection, J. Atmos. Sci. 71 (2014) $3767-3787$.

[69] M. Kurowski, W.W. Grabowski, and P.K. Smolarkiewicz, Anelastic and compressible simulations of moist dynamics at planetary scales, J. Atmos. Sci. 72 (2015) 3975-3995.

[70] M. Kurowski, D. Wojcik, M. Ziemianski, B. Rosa, Z. Piotrowski, Convectionpermitting regional weather modeling with COSMO-EULAG: Compressible and anelastic solutions for a typical westerly flow over the Alps, Month. Weather Rev. 144 (2016) 1961-1982. 
[71] P.H. Lauritzen, A Stability Analysis of Finite-Volume Advection Schemes Permitting Long Time Steps, Month. Weather Rev. 135 (2007) 26582673.

[72] P.H. Lauritzen, E. Kaas, B. Machenhauer, K. Lindberg, A mass-conservative version of the semi-implicit semi-Lagrangian HIRLAM, Q.J.R. Meteorol. Soc. 134 (2008) 15831595.

[73] A. Lapidus, Detached shock calculations by second-order finite differences, J. Comput. Phys. 2 (1967) 154-177.

[74] R. Laprise, Regional climate modelling, J. Comput. Phys. 277 (2008) 3641-3666.

[75] P. Lax, B. Wendroff, Systems of Conservation Laws, Commun. Pur. Appl. Math. 13 (1960) 217-237.

[76] C.E. Leith, Numerical simulation of the earth's atmosphere, in "Methods of Computational Physics" Vol 4, p.1, Eds., B. Alder, S. Ferenbach, M. Rotenberg, Academic Press (1965).

[77] A. Leonard, Dissipation of turbulence energy and scalar variance in large eddy simulations of turbulent flows, EOS T. Am. Geophys. 55 (1974) 137-138.

[78] R.J. Leveque, High resolution finite volume methods on arbitrary grids via wave propagation, J. Comput. Phys. 78 (1988) 36-63.

[79] X. Li, D. Chen, X. Peng, K. Takahashi, F. Xiao, A multimoment finite-volume shallow-water model on the Yin-Yang overset spherical grid Month. Weather Rev., 136 (2008), 3066-3086.

[80] X. Li, C. Chen, X. Shen, F. Xiao, A Multimoment Constrained Finite-Volume Model for Nonhydrostatic Atmospheric Dynamics, Month. Weather Rev. 141 (2013), 1216-1240.

[81] D.K. Lilly, On the numerical simulation of buoyant convection, Tellus 14 (1962) 148-172.

[82] D.K. Lilly, Introduction to "Computational Design for Long-Term Numerical Integration of the Equations of Fluid Motion: Two-Dimensional Incompressible Flow. Part I", J. Comput. Phys. 135 (1997) 101-102.

[83] P.F. Linden, J.M. Redondo, D.L. Youngs, Molecular mixing in Rayleigh-Taylor instability, J. Fluid Mech. 265 (1994) 97-124.

[84] F.B. Lipps, R.S. Hemler, A scale analysis of deep moist convection and some related numerical calculations, J. Atmos. Sci. 39 (1982) 2192-2210.

[85] F.B. Lipps, On the anelastic approximation for deep convection, J. Atmos. Sci. 47 (1990) 1794-1798.

[86] P. Lynch, The origins of computer weather prediction and climate modeling, J. Comput. Phys. 227 (2008) 3431-3444.

[87] R.W. MacCormack, A numerical methods for solving the equations of compressible viscous flows, AIAA J. 20 (1982) 1275-1281. 
[88] B. Machenhauer, E. Kaas, P.H. Lauritzen, Finite-volume methods in meteorology, in: R.Temam, J.Tribbia, P.Ciarlet (Eds.), Computational methods for the atmosphere and the oceans, Handbook of Numerical Analysis 14 (2009) 3-120, Elsevier.

[89] S. Malardel, N. Wedi, W. Deconinck, M. Diamantakis, C. Kühnlein, G. Mozdzynski, M. Hamrud, P. Smolarkiewicz, A new grid for the IFS, ECMWF Newsletter No. 146 (2016) 23-28.

[90] S. Malardel, N. Wedi, How does subgrid-scale parameterisation influence nonlinear spectral energy fluxes in global NWP models? J. Geophys. Res.-Atmos. 121 (2016) 5395-5410.

[91] L.G. Margolin, P.K. Smolarkiewicz, Z. Sorbjan, Large-eddy simulations of convective boundary layers using nonoscillatory differencing, Physica D 133 (1999) 390-397.

[92] L.G. Margolin, W.J. Rider, A Rationale for Implicit Turbulence Modeling, Int. J. Num. Meth. Fluids 39 (2002) 821-841.

[93] L.G. Margolin, P.K. Smolarkiewicz, A.A. Wyszogrodzki, Implicit turbulence modeling for high Reynolds number flows. J. Fluids Eng. 124 (2002) 862-867.

[94] L.G. Margolin, W.J. Rider, and F.F. Grinstein, J. Turbul. 7 N15 (2006) 1-27.

[95] S. Marras, J.F. Kelly, M. Moragues, A. Müller, M. Kopera, M. Vzquez, F. X. Giraldo, G. Houzeaux, O. Jorba, 2015: A Review of Element-Based Galerkin Methods for Numerical Weather Prediction: Finite Elements, Spectral Elements, and Discontinuous Galerkin, Archives of Computational Methods in Engineering, DOI 10.1007/s11831-015-9152-1.

[96] K. Maurin, Analysis Part I: Elements, Reidel Publ. Comp, Boston, 1976, 430 pp.

[97] M.J. Miller, P.K. Smolarkiewicz, Predicting weather, climate and extreme events (Preface), J. Comput. Phys. 227 (2008) 3429-3430.

[98] K. Morgan, J. Peiraire, J. Peiro, The computation of three-dimensional flows using unstructured meshes, Comput. Method. Appl. M. 87 (1991) 335-352.

[99] C.D. Munz, S. Roller, R. Klein, K.J. Geratz, The extension of incompressible flow solvers to the weakly compressible regime, Comput. Fluids 32 (2003) 173196.

[100] R.D. Nair, B. Machenhauer, The mass-conservative cell-integrated semiLagrangian advection scheme on the sphere, Month. Weather Rev. 130 (2002), 649667.

[101] R.D. Nair, J.S. Scroggs, F.H. Semazzi, A forward-trajectory global semiLagrangian transport scheme, J. Comput. Phys. 190 (2003) 275294.

[102] R.D. Nair, S.J. Thomas, R.D. Loft, A discontinuous Galerkin global shallow water model, Month. Weather Rev., 133 (2005), 876-888. 
[103] J. von Neumann, R.D. Richtmyer, A method for the numerical calculations of hydrodynamical shocks, J. Appl. Phys. 21 (1950) 232-237.

[104] N. Nikiforakis, Mesh generation and mesh adaptation for large-scale Earthsystem modelling, Introduction, Phil. Trans. R. Soc. A 367 (2009) 4473-4481.

[105] E.S. Oran, J.P. Boris, Computing turbulent shear flows - a convenient conspiracy, Comput. Phys. 7 (1993) 523-533.

[106] T. Palmer, Build high-resolution global climate models, Nature 515 (2014) 338-339.

[107] Z.P. Piotrowski, P.K. Smolarkiewicz, S.P. Malinowski, A.A. Wyszogrodzki, On numerical realizability of thermal convection, J. Comput. Phys. 228 (2009) 6268-6290.

[108] D.H. Porter, A. Pouquet, P.R. Woodward, Kolmogorov-like spectra in decaying three-dimensional supersonic flows, Phys. Fluids 6 (1994) 2133-2142.

[109] J.M. Prusa, P.K. Smolarkiewicz, An all-scale anelastic model for geophysical flows: dynamic grid deformation, J. Comput. Phys. 190 (2003) 601-622.

[110] J.M. Prusa, P.K. Smolarkiewicz, A.A Wyszogrodzki, EULAG, a computational model for multiscale flows, Comput. Fluids 37 (2008) 1193-1207.

[111] R.D. Richtmyer, K.W. Morton, Difference methods for initial-value problems, Interscience Publishers (1967) pp. 405.

[112] A.W. Rizzi, M. Inouye, Time-split finite-volume methods for three-dimensional blunt-body flow, AIAA J. 11 (1973) 1478-1485.

[113] R.B. Rood, Numerical advection algorithms and their role in atmospheric transport and chemistry models, Reviews of Geophysics, 25 (1987) 70-100.

[114] M. Satoh, T. Matsuno, H. Tomita, H. Miura, T. Nasuno, S. Iga, Nonhydrostatic icosahedral atmospheric model (NICAM) for global cloud resolving simulations, J. Comput. Phys. 227 (2008) 3486-3514.

[115] H. Schmidt, U. Schumann, Coherent structure of the convective boundary layer derived from large-eddy simulation, J. Fluid Mech. 200 (1989) 511-562.

[116] T. Schneider, N. Botta, K.J. Geratz, R. Klein, Extension of finite volume compressible flow solvers to multi-dimensional, variable density zero Mach number flows, J. Comput. Phys. 155 (1999) 248-286.

[117] Y. Seity, P. Brousseau, S. Malardel, G. Hello, P. Bénard, F. Bouttier, C. Lac, V Masson, The AROME-France convective-scale operational model, Month. Weather Rev. 139 (2011) 976-991.

[118] W.C. Skamarock, J.B. Klemp, A time-split nonhydrostatic atmospheric model for weather research and forecasting applications, J. Comput. Phys. 227 (2008) $3465-3485$. 
[119] W.C. Skamarock, J.B. Klemp, M.G. Duda, L.D. Fowler, S.-H. Park, T.D. Ringler, A multiscale nonhydrostatic atmospheric model using centroidal Voronoi tesselations and C-grid staggering, Month. Weather Rev. 140 (2012) 3090-3105.

[120] J. Smagorinsky, General circulation experiments with the primitive equations: I. The basic experiment, Month. Weather Rev. 91 (1963) 99-164.

[121] R.B. Smith, Linear theory of stratified flow past an isolated mountain in isosteric coordinates, J. Atmos. Sci. 45 (1988) 3889-3896.

[122] P.K. Smolarkiewicz, The multidimensional Crowley advection scheme, Month. Weather Rev. 113 (1997) 1968-1983.

[123] P.K. Smolarkiewicz, T.L. Clark, Numerical-simulation of the evolution of a 3-dimensional field of cumulus clouds. Part I: Model description, comparison with observations and sensitivity studies, J. Atmos. Sci. 42 (1985) 502-522.

[124] P.K. Smolarkiewicz, A fully multidimensional positive definite advection transport algorithm with small implicit diffusion, J. Comput. Phys., 54 (1984) $325-362$.

[125] P.K. Smolarkiewicz, R. Rotunno, Low Froude number flow past threedimensional obstacles. Part I: Baroclinically generated lee vortices, J. Atmos. Sci. 46 (1989) 1154-1164.

[126] P.K. Smolarkiewicz, J.A. Pudykiewicz, A class of semi- Lagrangian approximations for fluids, J. Atmos. Sci. 49 (1992), 2082-2096.

[127] P.K. Smolarkiewicz, L.G. Margolin, On forward-in-time differencing for fluids: extension to a curvilinear framework, Monthly Weather Rev. 121 (1993) 18471859 .

[128] P.K. Smolarkiewicz, L.G. Margolin, MPDATA: A finite-difference solver for geophysical flows, J. Comput. Phys. 140 (1998) 459-480.

[129] P.K. Smolarkiewicz, L.G. Margolin, A.A Wyszogrodzki, A class of nonhydrostatic global models, J. Atmos. Sci. 58 (2001) 349-364.

[130] P.K. Smolarkiewicz, J. Szmelter, Multidimensional positive definite advection transport algorithm (MPDATA): an edge-based unstructured-data formulation, Int. J. Numer. Meth. Fluids 47 (2005) 1293-1299.

[131] P.K. Smolarkiewicz, J. Szmelter, MPDATA: An edge-based unstructured-grid formulation, J. Comput. Phys. 206 (2005) 624-649.

[132] P.K. Smolarkiewicz, Multidimensional positive definite advection transport algorithm: an overview, Int. J. Numer. Meth. Fluids 50 (2006) 1123-1144.

[133] P.K. Smolarkiewicz, J. Szmelter, An MPDATA-based solver for compressible flows, Int. J. Numer. Meth. Fluids 50 (2006) 1123-1144.

[134] P.K. Smolarkiewicz, J. Szmelter, Iterated upwind schemes for gas dynamics, J. Comput. Phys. 228 (2009) 33-54. 
[135] P.K. Smolarkiewicz, J. Szmelter, A nonhydrostatic unstructured-mesh soundproof model for simulation of internal gravity waves, Acta Geophysica 59 (2011) 1109-1134.

[136] P.K. Smolarkiewicz, P. Charbonneau, EULAG, a computational model for multiscale flows: An MHD extension, J. Comput. Phys. 236 (2013) 608-623.

[137] P.K. Smolarkiewicz, J. Szmelter, A.A. Wyszogrodzki, An unstructured-mesh atmospheric model for nonhydrostatic dynamics, J. Comput. Phys. 254 (2013) 184-199.

[138] P.K. Smolarkiewicz, C. Kühnlein, N.P. Wedi, A consistent framework for discrete integrations of soundproof and compressible PDEs of atmospheric dynamics, J. Comput. Phys. 263 (2014) 185-205.

[139] P.K. Smolarkiewicz, W. Deconinck, M. Hamrud, C. Kühnlein, G. Mozdzynski, J. Szmelter, N.P. Wedi, A finite-volume module for simulating global all-scale atmospheric flows, J. Comput. Phys. 315 (2016) 287-304.

[140] S.T. Soong, Y. Ogura, A comparison between axisymmetric and slab symmetric cumulus models, J. Atmos. Phys. 30 (1973) 879-893.

[141] E.A. Spiegel, G. Veronis, On the Boussinesq approximation for compressible fluid, Astrophys. J. 131 (1960) 442-447.

[142] A. Staniforth and J.J. Côté, Semi-Lagrangian integration schemes for atmospheric models-A review, Month. Weather Rev. 119 (1991) 2206-2223.

[143] A. Staniforth, N. Wood, Aspects of the dynamical core of nonhydrostatic, deep-atmosphere, unified weather and climate-prediction model, J. Comput. Phys. 227 (2008) 3445-3464.

[144] A. Staniforth, J. Thuburn, Horizontal grids for global weather and climate prediction models: a review, Q.J.R. Meteorol. Soc. 138 (2012), 1-26.

[145] G.J. Steeneveld, R. Ronda, A.A.M. Holtslag, The challenge of forecasting the onset and development of radiation fog using mesoscale atmospheric models, Boundary-Layer Meteorol., 154 (2015) 265-289.

[146] J. Steppeler, R. Hess, U. Schättler, L. Bonaventura, Review of numerical methods for nonhydrostatic weather prediction models, Meteorol. Atmos. Phys. 82 (2003) 287-301.

[147] J. Szmelter, M.J. Marchant, A. Evans, N.P. Weatherill, Two-dimensional Navier-Stokes equations with adaptivity on structured meshes, Comput. Method. Appl. M. 101 (1992) 355-368.

[148] J. Szmelter, P.K. Smolarkiewicz, MPDATA error estimator for mesh adaptivity, Int. J. Numer. Meth. Fluids 50 (2006) 1269-1293.

[149] J. Szmelter, P. Ortiz, Burning surfaces evolution in solid propellants: a numerical model, P. I. Mech. Eng. G-J. Aer. 221 (2007), 429-440. 
[150] J. Szmelter, P.K. Smolarkiewicz, An edge-based unstructured mesh discretisation in geospherical framework, J. Comput. Phys. 229 (2010) 49804995 .

[151] J. Szmelter, P.K. Smolarkiewicz, An edge-based unstructured mesh framework for atmospheric flows, Comput. Fluids. 46 (2011) 455-460.

[152] J. Szmelter, Z. Zhang, P.K. Smolarkiewicz: An unstructured-mesh atmospheric model for nonhydrostatic dynamics: Towards optimal mesh resolution. J. Comput. Phys., 294 (2015), 363-381.

[153] M.C. Tapp, P.W. White, A non-hydrostatic mesoscale model. Q.J.R. Meteorol. Soc. 102 (1976) 277-296.

[154] M. Taylor, J. Tribbia, M. Iskandarani M, The spectral element method for the shallow water equations on the sphere, J. Comput. Phys. 130 (1997), 92-108.

[155] S.J. Thomas, R. Loft, The NCAR spectral element climate dynamical core: semi-implicit Eulerian formulation, J. Sci. Comput. 25 (2005), 307-322.

[156] J.F. Thompson, B.K. Soni, N.P. Weatherill, Handbook of Grid Generation, CRC Press, ISBN 0-8493-2687-7, (1999).

[157] J. Thuburn, Some conservation issues for the dynamical cores of NWP and climate models, J. Comput. Phys. 227 (2008) 3715-3730.

[158] J. Thuburn, C.J. Cotter, A primal-dual mimetic finite element scheme for the rotating shallow water equations on polygonal spherical meshes, J. Comput. Phys. 290 (2015) 274-297.

[159] E.F. Toro, Riemann Solvers and Numerical Methods for Fluid Dynamics, 3ed Edition, Springer (2010) $724 \mathrm{pp.}$

[160] C.J. Tremback, J. Powell, W.R. Cotton, R.A. Pileke, The forward-in-time upstream advection scheme: Extension to higher orders, Month. Weather Rev. 115 (1987) 540-555.

[161] E. Turkel, Review of preconditioning techniques for fluid dynamics, Appl. Numer. Math. 21 (1993) 257-284.

[162] P. Ullrich, C. Jablonowski, MCore: A non-hydrostatic atmospheric dynamical core utilizing high-order finite-volume methods, J. Comp. Phys., 231 (2012) 5078-5108.

[163] B. van Leer, Towards the ultimate conservative differences scheme I. The quest of monotonicity, Lecture Notes in Physics, 18 (1973) p. 163, Springer.

[164] B. van Leer, Towards the ultimate conservative difference scheme. V. A secondorder sequal to Godunov's method, J. Comput. Phys., 32 (1979) 101-136.

[165] M. Vinokur, Conservation equations of gasdynamics in curvilinear coordinate systems, J. Comput. Phys., 14 (1974), 105-125. 
[166] G. Volpe, Performance of compressible flow codes at low mach numbers, AIAA J. 31 (1993) 49-56.

[167] M.L. Waite, P.K. Smolarkiewicz, Instability and breakdown of vertical vortex pair in a strongly stratified fluid, J. Fluid. Mech. 606 (2008) 239-273.

[168] R.F. Warming, B.J. Hyett, The modified equation approach to the stability and accuracy analysis of finite-difference methods, J. Comput. Phys. 14 (1974) $159-179$.

[169] A. Warn-Varnas, J. Hawkins, P.K. Smolarkiewicz, S.A. Chin-Bing, D. King, Z. Hallock, Solitary wave effects north of Strait of Messina, Ocean Modelling 18 (2007) 97-121.

[170] N.P. Weatherill, Grid generation, Von Karman Lecture Notes, June 1989. 1-96, ISSN 0377-8312.

[171] N.P. Wedi, P.K. Smolarkiewicz, Extending Gal-Chen and Somerville terrainfollowing coordinate transformation on time dependent curvilinear boundaries, J. Comput. Phys. 193 (2004) 1-20.

[172] N.P. Wedi, P. Bauer, W. Deconinck, M. Diamantakis, M. Hamrud, C. Kühnlein, S. Malardel, K. Mogensen, G. Mozdzynski, P.K. Smolarkiewicz, The modelling indfrasturcture of the Integrated Forecasting System: Recent advances and future challenges, Technical Memorandum 760 (2015), ECMWF, pp. 48.

[173] N.P. Wedi, M. Hamrud, G. Mozdzynski, G. Austad, S. Curic, J. Bidlot, Global, non-hydrostatic, convection-permitting, medium-range forecasts: progress and challenges. ECMWF Newsletter No. 133 (2012) 17-22.

[174] M. Weiss, A. Smith, Preconditioning applied to variable and constant density flows, AIAA J. 33 (1995) 2050-2057.

[175] J. Whitaker, HIWPP non-hydrostatic dynamical core tests: Results from idealized test cases, (2015) www.nws.noaa.gov/ost/nggps/DycoreTestingFiles/HIWPP_idealized_tests-v8

[176] D. Williamson, Stability of difference approximations to certain partial differential equations of fluid dynamics, J. Comput. Phys. 1 (1966) 51-67.

[177] D.L. Williamson, The evolution of dynamical cores for global atmospheric models, J. Meteor. Soc. Japan 85B (2007) 241-268.

[178] J. Wu, J.Z. Zhu, J. Szmelter, O.C. Zienkiewicz, Error Estimation and Adaptivity in Navier-Stokes Incompressible Flows, Comput. Mech. 6 (1990) 259-270.

[179] F. Xiao, T. Yabe, Completely conservative and oscillationless semi-Lagrangian schemes for advection transportation J. Comput. Phys. 170 (2001), 498-522.

[180] F. Xiao, T. Yabe, X. Peng, H. Kobayashi, Conservative and oscillation-less atmospheric transport schemes based on rational functions, J. Geophys. Res., 107(D22) (2002), 4609, ACL 2-1-ACL 2-11. 
[181] F. Xiao, Unified formulation for compressible and incompressible flows by using multi-integrated moments I: one-dimensional inviscid compressible flow, J. Comput. Phys. 195 (2004) 629-654.

[182] F. Xiao, R. Akoh, S. Ii, Unified formulation for compressible and incompressible flows by using multi-integrated moments II: Multi-dimensional version for compressible and incompressible flows, J. Comput. Phys. 213 (2006) $31-56$.

[183] B. Xie, S. Ii, A. Ikebata, F. Xiao, A multi-moment finite volume method for incompressible Navier-Stokes equations on unstructured grids: Volumeaverage/point-value formulation, J. Comput. Phys. 277 (2014), 138-162.

[184] B. Xie, F. Xiao, Two and three dimensional multi-moment finite volume solver for incompressible Navier-Stokes equations on unstructured grids with arbitrary quadrilateral and hexahedral elements, Computers \& Fluids 104 (2014), 40-54.

[185] T. Yabe, P. Wang, Unified numerical procedure for compressible and incompressible fluid, J. Phys. Soc . Jpn. 60 (1991) 2105-2018.

[186] T. Yabe, F. Xiao, T. Utsumi, The constrained interpolation profile method for multiphase analysis, J. Comput. Phys. 169 (2001) 556-593.

[187] K.-S. Yeh, J. Côté, S. Gravel, A. Méthot, A. Patoine, M. Roch, A. Staniforth, The CMC-MRB global environmental multiscale (GEM) model. Part III: Nonhydrostatic formulation, Month. Weather Rev. 130 (2002) 339-356.

[188] M.P. Zhang, C.W. Shu, An analysis of and a comparison between the discontinuous Galerkin and the spectral finite volume methods, Comput. \& Fluids, 34 (2005) 581592.

[189] S.T. Zalesak, Fully multidimansional flux-corrected transport algorithms for fluids, J. Comput. Phys. 31 (1979) 335-362.

[190] M. Zerroukat, A simple mass conserving semi-Lagrangian scheme for transport problems, J. Comput. Phys. 229 (2010) 9011-9019.

[191] O.C. Zienkiewicz, J. Szmelter, J. Peraire, Compressible and incompressible flow; an algorithm for all seasons, Comput. Method. Appl. M. 78 (1990) 105121. 